\title{
Self-force on a scalar particle in spherically-symmetric spacetime via mode-sum regularization: radial trajectories
}

\author{
Leor Barack \\ Department of Physics, Technion-Israel Institute of Technology, Haifa, 32000, Israel
}

(October 26, 2018)

\begin{abstract}
Recently, we proposed a method for calculating the "radiation reaction" self-force exerted on a charged particle moving in a strong field orbit in a black hole spacetime. In this approach, one first calculates the contribution to the "tail" part of the self force due to each multipole mode of the particle's self field. A certain analytic procedure is then applied to regularize the (otherwise divergent) sum over modes. This involves the derivation of certain regularization parameters using local analysis of the (retarded) Green's function. In the present paper we present a detailed formulation of this mode-sum regularization scheme for a scalar charge on a class of static spherically-symmetric backgrounds (including, e.g., the Schwarzschild, Reissner-Nordström, and Schwarzschild-de Sitter spacetimes). We fully implement the regularization scheme for an arbitrary radial trajectory (not necessarily geodesic) by explicitly calculating all necessary regularization parameters in this case.
\end{abstract}

04.25-g, 04.30.Db, 04.70.Bw

\section{INTRODUCTION}

The motion of a test pointlike mass (a "particle") in orbit outside a black hole is commonly studied to model, and gain understanding of, realistic astrophysical scenarios involving highly relativistic two-body systems — particularly, the capture of a small compact object by a supermassive black hole [1]. To describe the orbital evolution of such a particle on a strongly curved background, one must take into account non-geodesic effects caused by the interaction of the particle with its own gravitational field. This problem of deducing the self force (or "radiation reaction" force) exerted on the particle is often treated via perturbation theory: one assumes that the particle is endowed with a charge $q$ much smaller than the mass of the black hole (this charge may represent the particle's mass, electric charge or - as in the toy model studied in the current paper - its scalar charge) and looks for the $O\left(q^{2}\right)$ correction to the equation of motion. The basic challenging task involved in this calculation, already in flat space, is, of course, correctly handling the divergence of the self field at the very location of the particle; namely, the introduction and justification of an appropriate regularization method. When considering the case of curved spacetime, additional difficulty arises due to the nonlocal nature of the self-force effect: waves emitted by the particle at some moment may backscatter off spacetime curvature and interact back with the particle at later stages of its motion. The occurrence of this so-called "tail" contribution to the self force results in that the calculation of the self force at a given moment requires, in principle, knowledge of the entire causal history of the particle. A number of methods (briefly surveyed below) have been proposed over the years for calculating the self force in curved backgrounds. The interest in this problem has greatly risen lately by virtue of recent years' developments towards experimental gravitational waves detection, and the consequent need for accurate predictions for the orbital evolution of strongly gravitating two-body systems. Yet, actual calculations of the self force have been restricted thus far only to a few very simple cases (see below).

The standard technique for calculating the radiative evolution of orbits around black holes is the one based on energy-momentum balance considerations [2]. In this approach one computes the flux to infinity (and across the horizon) of quantities associated with the constants of motion in the lack of self-force effects (specifically, in the Schwarzschild and Kerr backgrounds, the particle's energy $E$ and azimuthal angular momentum $L_{z}$ ), thus deducing the temporal rate of change of these "constants". Such balance calculations, though developed to a great extent, present two basic drawbacks: First, in the important Kerr case they are inapplicable for calculating the rate of change of the third constant of motion necessary for a full specification of the orbital evolution, i.e., the Carter constant $Q$, as this quantity is not additive. Second, these calculations do not account for the non-dissipative, yet important, part of the self force [3,, .

For the above reasons, a method based on direct calculation of the momentary force along the worldline seems more adequate. In the context of electromagnetism in flat space, such a method is familiar from the classic work by Dirac [5], concerning the electromagnetic self force on a (classical) electron. Dirac avoided the singularity of the self field at the particle's location by introducing the "radiative potential", constructed by taking the difference between the retarded and advanced electromagnetic potentials (what results in the cancellation of the problematic singular part). 
This procedure gave rise to what is now called the Abraham-Lorentz-Dirac (ALD) self force in flat space [see Eq. (10) below for the analogous scalar particle case]. The concept of "radiative potential" was much later employed by Gal'tsov [6] for calculating the temporal rate of change of the energy and azimuthal angular momentum parameters for electrically charged particles orbiting a Kerr black hole. Though Gal'tsov analysis yielded correct results, it seems conceptually difficult, in general cases, to justify the use of such a non-causal approach. The problem becomes obvious when considering curved spacetime, where the self force exhibits nonlocal contributions: according to this approach, the force acting on a particle at a given moment turns out to be affected by the entire future evolution of the particle.

A formal method for calculating the momentary self force in curved spacetime, which employs the purely retarded Green's function and is thus inherently causal, was developed long ago by DeWitt and Brehme [7] (for the electromagnetic case). Dewitt and Brehme first carried out a local analysis of the retarded Green's function near the particle's worldline, based on the Hadamard expansion [8] (which is, basically, an expansion of the Green's function in powers of the geodesic distance between the source and evaluation points). Then, the particle's equation of motion was deduced by imposing local energy-momentum conservation on a thin world-tube surrounding the particle's worldline. Following the same approach (though using a different renormalization scheme), Mino, Sasaki, and Tanaka [9] studied the gravitational self force by analyzing the local metric perturbations near a particle. They concluded that the regularized gravitational self force in vacuum is due solely to a nonlocal tail contribution. It remained unclear, though, how to practically evaluate the formal expression derived for this tail (see, however, recent attempts to tackle this problem [10]).

Recently, Quinn and Wald [11] developed a different, axiomatic, approach to the regularization problem of the self force in curved spacetimes. Their approach relies on a "comparison axiom", which allows the calculation of the self force by comparing the given problem with a (properly chosen) analogous problem in flat space (see 11] for details). The implementation of this approach for both the electromagnetic and the gravitational cases [11] yielded formal results in agreement with those obtained by DeWitt and Brehme (as corrected by Hobbs [12]; the main result by DeWitt and Brehme contained a trivial error) and by Mino et al., with the advantage of involving much simpler calculations. More recently, the same approach was applied by Quinn [13] for a scalar particle [the main results of this work, also quoted in Ref. [14, are summarized below; see Eqs. (9)-(13)]. Again, however, despite the availability of a simple formal framework for obtaining equations of motion for a test particle in curved spacetime, the practical implementation of the formalism in actual calculations (particularly, the evaluation of the nonlocal tail contribution) remained a challenging task.

So far, the study of the self force effect in concrete situations have been restricted to very few simple cases. DeWitt and DeWitt [15] employed the above mentioned formalism by DeWitt and Brehme to study the self force correction to the geodesic equation for an electrically charged particle freely falling in a static weak gravitational field, in the limit of small velocity. They concluded that a repulsive force of $\propto q^{2} r^{-3}$ magnitude (where $r$ is the Schwarzschild radial coordinate) would be experienced by such a particle, in addition to the usual attractive inverse-square force. Later, Smith and Will [16] (and, independently, Frolov and Zel'nikov [17]) were able to derive an exact analytic expression for the $O\left(q^{2}\right)$ self force acting on an electrically charged particle held static in the Schwarzschild exterior. They found a repulsive self force of exact magnitude $M q^{2} r^{-3}$ [measured by a momentarily static freely falling observer, and expressed in relativistic (geometrized) units]. This result was later extended by Frolov and Zel'nikov [18] to scalar and electrically-charged particles held static outside a charged, Reissner-Nordström type, black hole. It was concluded that, unlike in the electromagnetic case, no self force is experienced by a static scalar particle. This last result has been reproduced very recently by Wiseman [3 in a thorough analysis of the self force acting on a static scalar particle in Schwarzschild spacetime.

The exact calculation in the static electric charge case was made possible owing to the existence of an exact analytic solution, discovered long ago by Copson [19] (and later corrected by Linet [20]), for the electrostatic potential of a static charge in the Schwarzschild geometry. (The analogous closed-form solution for a scalar particle was constructed by Wiseman in [3].) In more general cases one cannot benefit from the existence of such exact solutions. The usual approach for treating this problem, of obtaining solutions to the field equation in black hole backgrounds, is through the Fourier-multipole decomposition of the field. In the context of the radiation reaction problem this approach seems to offer two obvious advantages: First, it allows, in the usual manner, reduction of the field equation [originally a partial differential equation (DE) in $1+3$ dimensions] to an ordinary DE, thus making it accessible to simple numerical treatment. Second, each individual mode of the field turns out to be continuous (and the corresponding self force to be bounded) even at the particle's location. Having this in mind, Ori previously proposed [21] that a practical calculation of the self force effect may be carried out by first evaluating the effect of each Fourier-multipole mode of a particle's self retarded field on its radiative evolution (through the local self force experienced by the particle), and then summing over all modes.

In Ref. 21] the above sum-over-modes approach has been successfully applied for the calculation of the adiabatic, 
orbit-integrated, evolution rate of the three constants of motion in Kerr spacetime, including the Carter constant.|T However, it appears that a naive application of this method for the calculation of the momentary self force would not be useful, in general. The reason is that, although each mode yields a finite contribution to the self force, the sum over all modes is found, in general, to diverge. This situation manifests itself already in the most simple case, that of a static scalar charge in flat space: in this basic example, the contribution of each multipole mode to the radial component of the self force is the same, $-q^{2} /\left(2 r_{0}^{2}\right)$ (where $r_{0}$ is the distance of the particle from the origin of coordinates, with respect to which the spherical harmonic functions are defined), with an obvious divergence of the sum over modes. This, however, does not mean that one has to abandon the whole sum-over-modes approach; one may still be able to benefit from its advantages, by introducing a suitable regularization procedure into the calculation, properly designed to overcome the above kind of divergence.

In a previous paper 22 we introduced the basic elements of a method for the calculation of the self force in curved spacetime through regularization of the mode sum. The implementation of the proposed calculation method for a specific trajectory in a given spacetime consists of two stages. First, one solves (numerically, in most cases) the appropriate ordinary DE for each Fourier-harmonic mode $l, m, \omega$ of the retarded field, and evaluates the (finite) contribution of each of these modes to the self force. (Alternatively, one may numerically solve the $1+1$ partial DE in the time domain, for each multipole mode $l, m$.) Then, the sum over all modes is made subject to a certain regularization procedure, which requires the knowledge of several regularization parameters. These parameters are derived analytically, for any given trajectory, through local perturbative analysis of the (retarded) Green's function. In Ref. 22] we outlined this regularization method as applied to a scalar particle moving on a Schwarzschild background, and presented final results (i.e., the values of all necessary regularization parameters) for the case of radial motion. The target of the present paper is three-fold: (i) providing a systematic presentation of the regularization scheme (including a discussion of some mathematical subtleties left untreated in 22]); (ii) providing full details of the calculations involved in deriving the regularization parameters for radial trajectories; and (iii) extending the analysis to a wider class of static spherically-symmetric black hole spacetimes.

This paper (as well as Ref. [22]) is concerned with the analytic part of the regularization scheme; namely, it sets the mathematical foundation for the scheme, and demonstrates the calculation of the regularization parameters involved in its implementation (in the example of radial motion). As explained above, full calculation of the self force requires the supplementary numerical determination of the various modes' "bare" contributions to the self force. This was recently done for various trajectories of a scalar particle outside a Schwarzschild black hole: Burko first analyzed the case of a static particle [23] and the one of a particle in circular motion [24](see also [25]). More recently, Barack and Burko applied the regularization scheme for studying radial trajectories in Schwarzschild [26]. These numerical works confirm the applicability of the regularization scheme, and provide support for the values of the analytically deduced regularization parameters. Of course, they also yield significant physical information. In the static scalar particle case, Burko recovered the familiar result, of a zero self force. Calculations of the self force on a scalar particle in circular and radial trajectories were carried out for the first time (see Refs. [23,24,26] for details).

The current paper is organized as follows: In Sec. [1] we give some preliminary relations involving the self field, the Green's function, and the self force for a scalar particle. In Sec. III we decompose the Green's function into its spherical harmonic components, and discuss the applicability of this expansion. In Sec. IV we decompose the (tail part of the) self force into its spherical harmonic contributions, discuss the need for regularization of the mode sum, and present the regularization scheme. The implementation of this scheme involves local analysis of the Green's function modes for large multipole numbers, which is carried out in Sec. V. The particular case of radial motion is then considered in Secs. VI and VII, where the regularization parameters for this case are explicitly calculated. In Sec. VIII we summarize, discuss possible extensions of the analysis, and briefly survey some related work.

\section{SELF FORCE ON A SCALAR CHARGE: PRELIMINARIES}

We consider a class of static spherically-symmetric (not necessarily vacuum) black hole geometries, having a line element of the formp

$$
d s^{2}=-f(r) d t^{2}+f^{-1}(r) d r^{2}+r^{2}\left(d \theta^{2}+\sin ^{2} \theta d \varphi^{2}\right)
$$

\footnotetext{
${ }^{1}$ However, whereas the mode sum for the evolution rate of the energy and azimuthal angular momentum parameters was shown to converge 21], it is not clear yet whether the corresponding mode sum for the Carter constant converges or not.

${ }^{2}$ Throughout this paper we use relativistic units (with $G=c=1$ ), and metric signature -+++ .
} 
where $t, r, \theta$, and $\varphi$ are the Schwarzschild coordinates, and $f$ is a function of $r$ only, positive outside the event horizon. Important members of this class include the Schwarzschild solution, with $f=1-2 M / r$; the ReissnerNordström solution, with $f=1-2 M / r+Q / r^{2}$; and the Schwarzschild-de Sitter solution, with $f=1-2 M / r+\Lambda r^{2} / 3$. Here, $M$ stands for the black hole's mass, $Q$ represents its net electric charge, and $\Lambda$ is the cosmological constant.

We next consider a point-like particle of scalar charge $q$, with $|q| \ll M$, moving in a spacetime of the above type. Let $x^{\mu}=x_{p}^{\mu}(\tau)$ represent the particle's worldline (not necessarily a geodesic), with $\tau$ being its proper time. The scalar particle exhibits a Klein-Gordon field $\Phi$, satisfying

$$
\square \Phi \equiv \Phi_{; \alpha}^{; \alpha}=\frac{1}{\sqrt{-g}}\left(\sqrt{-g} g^{\alpha \beta} \Phi_{, \alpha}\right)_{, \beta}=-4 \pi \rho\left(x^{\mu}\right),
$$

where $\square$ represents the covariant D'Alembertian operator, $g$ is the metric determinant, and $\rho\left(x^{\mu}\right)$ is the scalar charge density, given by

$$
\rho\left(x^{\mu}\right)=q \int_{-\infty}^{\infty} \frac{1}{\sqrt{-g}} \delta^{4}\left(x^{\mu}-x_{p}^{\mu}(\tau)\right) d \tau .
$$

The solution for the scalar field can be written as

$$
\Phi\left(x^{\mu}\right)=\int G\left(x^{\mu} ; x^{\prime \mu}\right) \rho\left(x^{\prime \mu}\right) \sqrt{-g} d^{4} x^{\prime},
$$

where $G\left(x^{\mu} ; x^{\prime \mu}\right)$ is the retarded Green's function, satisfying

$$
\square G\left(x^{\mu} ; x^{\prime \mu}\right)=\frac{-4 \pi}{\sqrt{-g}} \delta^{4}\left(x^{\mu}-x^{\prime \mu}\right),
$$

and subject to the causality condition, $G=0$ whenever $x^{\mu}$ lies outside the future light cone of $x^{\prime \mu}$. Combining Eqs. (3) and (4) we obtain for the scalar field

$$
\phi\left(x^{\mu}\right)=q \int_{-\infty}^{\infty} G\left[x^{\mu} ; x_{p}^{\mu}(\tau)\right] d \tau
$$

The "scalar force" experienced by the particle due to its own field shall be taken, following [14], to be

$$
F_{\alpha}=q \Phi_{; \alpha}=q \Phi_{, \alpha}
$$

evaluated at the particle's location. We comment that the so-defined force is not perpendicular to the four-velocity of the particle, $u^{\alpha} \equiv d x_{p}^{\alpha}(\tau) / d \tau$, resulting in that the mass parameter of the particle is not conserved along the worldline. Indeed, the force on a scalar particle can be calculated otherwise (as in [6], e.g.), such as to make the mass parameter conserved: $F_{\alpha}^{\perp}=q\left(\Phi_{, \alpha}+u_{\alpha} u^{\beta} \Phi_{, \beta}\right)$. Although we shall adopt here the simpler definition, Eq. (7), the results of our analysis could then easily be applied for the force $F_{\alpha}^{\perp}$ as well. (Given all vectorial components of $F_{\alpha}$, one can easily construct both the force component perpendicular to the worldline, and the component tangent to it.) With Eq. (6) we now have for the self force acting on the particle at a point $x_{0}^{\mu} \equiv x_{p}^{\mu}(\tau=0)$ along its worldline,

$$
F_{\alpha}=q^{2} \int_{-\infty}^{\infty} G_{, \alpha}\left[x^{\mu} ; x_{p}^{\mu}(\tau)\right] d \tau
$$

where the gradient (taken with respect to $x^{\mu}$ ) is to be evaluated at $x^{\mu}=x_{0}^{\mu}$.

The "bare" self force given in Eq. (8) needs to be regularized to avoid divergences associated with the behavior of the scalar field at the very location of the particle. For that goal, Quinn [13, 14 applied the "comparison axiom" approach by Quinn and Wald [1] for the scalar particle case. The total self force acting on the scalar particle was found to be composed of three parts:

$$
F_{\alpha}^{(\text {total })}=F_{\alpha}^{(\mathrm{ALD})}+F_{\alpha}^{(\mathrm{Ricci})}+F_{\alpha}^{(\text {tail })}
$$

The first term here is a local ALD-like term, reading

$$
F_{\alpha}^{(\mathrm{ALD})}=\frac{1}{3} q^{2}\left(\dot{a}_{\alpha}-a^{2} u_{\alpha}\right)
$$


where $a^{\alpha}$ is the four-acceleration of the particle, $a^{2} \equiv a_{\beta} a^{\beta}$, and an overdot represents covariant differentiation with respect to the particle's proper time $\tau$. The second term in Eq. (9) is related to the local Ricci curvature at the particle location. It is given by

$$
F_{\alpha}^{(\mathrm{Ricci})}=\frac{1}{6} q^{2}\left(R_{\alpha \beta} u^{\beta}+u_{\alpha} R_{\beta \gamma} u^{\beta} u^{\gamma}-R u_{\alpha} / 2\right),
$$

where $R_{\alpha \beta}$ is the Ricci tensor and $R$ is the curvature scalar. The third term in the expression for the total self force represents the non-local "tail" contribution. It may be expressed as

$$
F_{\alpha}^{(\text {tail })} \equiv \lim _{\epsilon \rightarrow 0^{+}} F_{\alpha}^{(\epsilon)}
$$

where

$$
F_{\alpha}^{(\epsilon)} \equiv q^{2} \int_{-\infty}^{-\epsilon} G_{, \alpha}\left[x_{0}^{\mu} ; x_{p}^{\mu}(\tau)\right] d \tau
$$

As we mentioned above, the occurrence of a tail term - a prominent feature of the self force in curved spacetimes is due to the Green's function having its support also inside the source's future light cone. From the physical point of view, this is associated with the fact that waves are scattered off spacetime curvature while propagating on a curved background.

The task of implementing the formal expression, Eq. (9), in practical calculations of the self force is a challenging one. The difficulty stems, of course, from the need to evaluate the tail part, which requires knowledge of the Green's function everywhere along the particle's past worldline. Below we therefore focus on the tail term contribution to the self force, presenting a practical method for its calculation.

\section{MULTIPOLE DECOMPOSITION OF THE GREEN'S FUNCTION}

The regularization scheme to be introduced below is based on evaluating the contribution to the (tail part of the) self force due to each multipole mode of the (retarded) Green's function. To that end we first consider the multipole decomposition of the Green's function.

To begin, one may be tempted to decompose $G$ into its multipole modes $G^{l}$ in the usual manner, as $G=\sum_{l=0}^{\infty} G^{l}$ (where $G^{l}$ represents the quantity resulting from summing over azimuthal numbers $m$ ). Although this may look as standard procedure, caution is necessary here: in general, such a decomposition turns out ill-defined, as the sum over $l$ is found to diverge. This can be illustrated already in flat space. In this case, the modes $G^{l}$ admit a closed-form expression, which, for evaluation point $x^{\mu}$ lying inside the future light cone of the source point $x^{\prime \mu}$, is given by

$$
G_{\text {Flat }}^{l}\left(x^{\mu}, x^{\prime \mu}\right)=\frac{(2 l+1) P_{l}(\cos \chi) P_{l}\left[1-\sigma /\left(r r^{\prime}\right)\right]}{2 r r^{\prime}} .
$$

Here, $P_{l}$ is the Legendre polynomial, $\sigma \equiv \frac{1}{2}\left[\left(t-t^{\prime}\right)^{2}-\left(r-r^{\prime}\right)^{2}\right]$, and

$$
\cos \chi \equiv \cos \theta \cos \theta^{\prime}+\sin \theta \sin \theta^{\prime} \cos \left(\varphi-\varphi^{\prime}\right) .
$$

[Eq. (14) can be verified by direct substitution, using Eqs. (27), (28), and (30), to be given below.] Consider, for example, the case $\chi=0$, with $P_{l}(\cos \chi) \equiv 1$ for all $l$, corresponding to both the source and evaluation points lying in the same radial direction. At large $l$ values, the Legendre polynomial $P_{l}(\sigma)$ admits the asymptotic form $\propto l^{-1 / 2} \times$ oscillations with respect to $l$ [the exact asymptotic form is given in App. A below; see Eq. (A13)]. Thus, at large $l$ one finds $G_{\text {Flat }}^{l} \propto l^{1 / 2} \times$ oscillations, implying that the infinite sum over all modes fails to converge $]^{3}$ Mathematically speaking, this failure of the naive multipole decomposition may be associated with the fact that the Green's function of our problem exhibits a strong irregularity along the intersection of the future light cone of the source with the sphere of constant $r$ and $t$. In Appendix A we discuss this irregularity in more detail, referring to the analysis by DeWitt and Brehme [7]. We then suggest a way to overcome the difficulty caused by the presence of the irregularity,

\footnotetext{
${ }^{3}$ In the more general case, with $\chi \neq 0$, there is also a $\propto l^{-1 / 2} \times$ oscillations factor coming at large $l$ from $P_{l}$ (cos $\chi$ ), yielding the asymptotic form $G_{\text {Flat }}^{l} \propto$ const $\times$ oscillations. Hence, clearly, the sum over modes $G^{l}$ diverges in the general case as well.
} 
and explain how a well defined mode decomposition can still be accomplished. Although the detailed discussion of this issue is left to the Appendix, we outline here the basic argument, and present some definitions and notation needed in the sequel.

In Appendix A we construct a "modified" Green's function $G_{\bmod }=G-\delta G$, where the function $\delta G$ is chosen such that $G_{\text {mod }}$ has the following properties: (i) it is a continuous function of $\theta$ and $\varphi$ across the sphere of constant $r$ and $t$; and (ii) it yields the same self force, through Eq. (13), as the original Green's function $G$ (this is guaranteed by taking the function $\delta G$ to have no support inside the future light cone of the source). It is then argued that the modified Green's function admits an (absolutely) convergent multipole expansion, $G_{\bmod }=\sum_{l}\left(G^{l}-\delta G^{l}\right)$. Next, we define the new operation $\widetilde{\lim }_{l \rightarrow \infty}$ ("tilde-limit") of a series of numbers $A_{l}$, as the standard $\operatorname{limit}_{l}$ lim $\rightarrow \infty$ (when existing and finite) of the series $A_{l}-B_{l}^{(1)}-B_{l}^{(2)} \cdots-B_{l}^{(k)}$, where the $B_{l}^{(j)}$ 's are any finite number of terms having the form $B_{l}^{(j)}=a_{j} l^{b_{j}} \cos \left(\alpha_{j} l+\beta_{j}\right)$, with $a_{j}, b_{j}, \alpha_{j}$, and $\beta_{j}$ being some $l$-independent real numbers, and with none of the numbers $\alpha_{j}$ vanishing. Namely, if there exist $k$ quantities $B_{l}^{(j)}$ of the above form, such that subtracting them from the original series $A_{l}$ would yield a well-defined finite limit as $l \rightarrow \infty$, then we define

$$
\widetilde{\lim }_{l \rightarrow \infty} A_{l} \equiv \lim _{l \rightarrow \infty}\left[A_{l}-\sum_{j=1}^{k} B_{l}^{(j)}\right] .
$$

We also define the "tilde-sum" of a series $A_{l}$ by

$$
\sum_{l=0}^{\widetilde{\infty}} A_{l} \equiv \widetilde{\lim }_{\bar{l} \rightarrow \infty} \sum_{l=0}^{\bar{l}} A_{l}
$$

where $\sum_{l}^{\bar{l}}$ is the standard summation operation. It can be easily verified (see App. A that when the "tilde-limit" (or the "tilde-sum") of a series exists, then it is unique. In particular, if a standard infinite sum $\sum_{l}^{\infty} A_{l}$ converges, then one may replace it with a "tilde-sum" operation. Thus, we may replace the convergent standard sum $G_{\text {mod }}=$ $\sum_{l=0}^{\infty}\left(G^{l}-\delta G^{l}\right)$ with a tilde-summation. In Appendix $\mathrm{A}$ we show that $\sum_{l=0}^{\widetilde{\infty}}\left(\delta G^{l}\right)=0$. Consequently, we conclude

$$
G_{\text {mod }}=\sum_{l=0}^{\widetilde{\infty}} G^{l} .
$$

We emphasize once more that the modified Green's function $G_{\bmod }$ can serve instead of the original function $G$ for the calculation of self force, as both functions yield the same force. Eq. (18) thus implies that the calculation of the self force can be carried out through analysis of the original Green's function's modes $G^{l}$, by applying the tilde-summation instead of the (ill-defined) standard summation. (A more thorough discussion of the arguments leading to Eq. (18) will be given in Appendix A.)

Let us now turn to study the form of the multipole modes $G^{l}$ in greater detail. These modes can be written more explicitly as

$$
G^{l}\left(x^{\mu}, x^{\prime \mu}\right)=\sum_{m=-l}^{l} Y_{l m}(\theta, \varphi) \hat{g}^{l m}\left(t, r ; x^{\prime \mu}\right),
$$

where $Y^{l m}(\theta, \varphi)$ are the standard spherical harmonic functions on the sphere of constant $r$ and $t$. Substituting Eq. (19) and the relation $\delta\left(\theta-\theta^{\prime}\right) \delta\left(\varphi-\varphi^{\prime}\right) / \sin \theta=\sum_{l, m} Y_{l m}(\theta, \varphi) Y_{l m}^{*}\left(\theta^{\prime}, \varphi^{\prime}\right)$, (where an asterisk denotes complex conjugation) in Eq. (5), we obtain from the orthogonality of the spherical harmonics,

$$
r^{2} f^{-1}(r) \hat{g}_{, t t}^{l m}-\left[r^{2} f(r) \hat{g}_{, r}^{l m}\right]_{, r}+l(l+1) \hat{g}^{l m}=4 \pi \delta\left(t-t^{\prime}\right) \delta\left(r-r^{\prime}\right) Y_{l m}^{*}\left(\theta^{\prime}, \varphi^{\prime}\right)
$$

In terms of the $m$-independent variable $\tilde{g}^{l}\left(t, r ; t^{\prime}, r^{\prime}\right)$, defined through

$$
\hat{g}^{l m}=2 \pi \tilde{g}^{l} Y_{l m}^{*}\left(\theta^{\prime}, \varphi^{\prime}\right) /\left(r r^{\prime}\right),
$$

Eq. (20) becomes

$$
\tilde{g}_{, t t}^{l}-\tilde{g}_{,_{*} r_{*}}^{l}+4 V^{l}(r) \tilde{g}^{l}=2 f(r) \delta\left(t-t^{\prime}\right) \delta\left(r-r^{\prime}\right)
$$


where the radial coordinate $r_{*}(r)$ admits $d r_{*} / d r=f^{-1}(r)$, and the effective potential $V^{l}(r)$ is given by

$$
V^{l}(r)=\frac{1}{4} f(r)\left(\frac{l(l+1)}{r^{2}}+\frac{f^{\prime}(r)}{r}\right)
$$

(with a prime denoting $d / d r$ ).

To account for the causality condition, it is convenient to introduce the (Eddington-Finkelstein-like) null coordinates

$$
v \equiv t+r_{*} \quad \text { and } \quad u \equiv t-r_{*} .
$$

The relation $\delta\left(t-t^{\prime}\right) \delta\left(r-r^{\prime}\right)=2 f^{-1}\left(r^{\prime}\right) \delta\left(v-v^{\prime}\right) \delta\left(u-u^{\prime}\right)$ can then be used to write Eq. (22) in the simple form

$$
\tilde{g}_{, v u}^{l}+V^{l}(r) \tilde{g}^{l}=\delta\left(v-v^{\prime}\right) \delta\left(u-u^{\prime}\right) .
$$

We now impose causality by writing

$$
\tilde{g}^{l}=g^{l}\left(v, u ; v^{\prime}, u^{\prime}\right) \Theta\left(v-v^{\prime}\right) \Theta\left(u-u^{\prime}\right),
$$

where $\Theta$ is the standard step function. The "reduced Green's function" $g^{l}\left(v, u ; v^{\prime}, u^{\prime}\right)$ obeys the homogeneous equation

$$
g_{, v u}^{l}+V^{l}(r) g^{l}=0
$$

for all $u>u^{\prime}$ and $v>v^{\prime}$. Substituting Eq. (26) into Eq. (25) and examining the behavior along the null rays $v=v^{\prime}$ and $u=u^{\prime}$, one finds that $g^{l}$ must admit

$$
g^{l}\left(v=v^{\prime}\right)=g^{l}\left(u=u^{\prime}\right)=1 .
$$

For any fixed source point $v^{\prime}, u^{\prime}$, the homogeneous equation (27), supplemented by the initial conditions (28), constitutes a characteristic initial-value problem for the function $g$ anywhere at $u>u^{\prime}$ and $v>v^{\prime}$.

Finally, to express $G^{l}$ in terms of the reduced Green's function $g^{l}$, we substitute Eq. (21) [with Eq. (26)] into Eq. (19). In the resulting expression we can explicitly sum over $m$ by making use of the relation 28]

$$
\sum_{m=-l}^{l} Y_{l m}(\theta, \varphi) Y_{l m}^{*}\left(\theta^{\prime}, \varphi^{\prime}\right)=(4 \pi)^{-1}(2 l+1) P_{l}(\cos \chi),
$$

where $\cos \chi$ is the quantity given in Eq. (15). We then find for the $l$-mode of the Green's function,

$$
G^{l}=L P_{l}(\cos \chi) \frac{g^{l}\left(v, u ; v^{\prime}, u^{\prime}\right)}{r r^{\prime}} \Theta\left(v-v^{\prime}\right) \Theta\left(u-u^{\prime}\right)
$$

where we have set

$$
L \equiv l+1 / 2 .
$$

\section{MODE SUM REGULARIZATION SCHEME}

\section{A. The need for a mode sum regularization}

Following the discussion of the preceding section, we now replace $G$ in Eq. (13) by $G_{\text {mod }}$, and then substitute for $G_{\text {mod }}$ from Eq. (18). We find

\footnotetext{
${ }^{4}$ It is assumed here that both the differentiation and the integration involved in constructing $F_{\alpha}^{(\epsilon)}$ out of $G$ can be performed term-by-term with respect to the tilde-summation. This assumption should be verified by more closely inspecting the convergence properties of the tilde-sum over $G^{l}$ in Eq. (18), which, however, would be beyond the scope of the current paper.
} 


$$
F_{\alpha}^{(\epsilon)}=\sum_{l=0}^{\widetilde{\infty}} F_{\alpha}^{l(\epsilon)},
$$

where $F_{\alpha}^{l(\epsilon)}$ represents the contribution to $F_{\alpha}^{(\epsilon)}$ associated with the $l$-mode Green's function:

$$
F_{\alpha}^{l(\epsilon)}=q^{2} \int_{-\infty}^{-\epsilon} G_{, \alpha}^{l}\left[x_{0}^{\mu} ; x_{p}^{\mu}(\tau)\right] d \tau .
$$

For practical reasons which become clear below, let us now write

$$
F_{\alpha}^{l(\epsilon)}=F_{\alpha}^{l}-\delta F_{\alpha}^{l(\epsilon)},
$$

in which

$$
F_{\alpha}^{l}=q^{2} \int_{-\infty}^{\infty} G_{, \alpha}^{l}\left[x_{0}^{\mu} ; x_{p}^{\mu}(\tau)\right] d \tau \quad \text { and } \quad \delta F_{\alpha}^{l(\epsilon)}=q^{2} \int_{-\epsilon}^{\infty} G_{, \alpha}^{l}\left[x_{0}^{\mu} ; x_{p}^{\mu}(\tau)\right] d \tau
$$

Here, $F_{\alpha}^{l}$ is the $l$-mode of $F_{\alpha}=q \Phi_{, \alpha}$-the quantity given in Eq. (8), which is sourced by the entire worldline. This quantity can be obtained from the $l$-mode of the self field, which, in turn, can be calculated essentially with no difficulty (using numerical methods, in most cases [23,24,26]). The other quantity appearing in Eq. (34), $\delta F_{\alpha}^{l(\epsilon)}$, is local in nature, and thus may be treated, in principle, by means of local analytic methods (as we, indeed, demonstrate in this paper).

In terms of $F_{\alpha}^{l}$ and $\delta F_{\alpha}^{l(\epsilon)}$, the tail part of the self force is calculated through

$$
F_{\alpha}^{(\text {tail })}=\lim _{\epsilon \rightarrow 0^{+}} \sum_{l=0}^{\widetilde{\infty}}\left(F_{\alpha}^{l}-\delta F_{\alpha}^{l(\epsilon)}\right) .
$$

To carry out this calculation, one may tempt to first calculate the sum over $F_{\alpha}^{l}$ (which is $\epsilon$-independent), and then evaluate the local contribution $\lim _{\epsilon \rightarrow 0^{+}} \widetilde{\sum}_{l} \delta F_{\alpha}^{l(\epsilon)}$. However, here one comes across a problem: Although each of the modes $F_{\alpha}^{l}$ yields a finite contribution at the particle's location, in general the sum over all modes $F_{\alpha}^{l}$ diverges. As we mentioned in the Introduction, this can be demonstrated even in the simple case of a static scalar charge in flat space. To overcome this type of divergence, the introduction of a certain regularization procedure for the mode sum is required. Such a procedure is described (and later implemented) in what follows.

\section{B. The regularization scheme}

To regularize the modes sum, one seeks a (simple as possible) $\epsilon$-independent function $h_{\alpha}^{l}$, such that the series $\sum_{l}\left(F_{\alpha}^{l}-h_{\alpha}^{l}\right)$ would converge. Once such a function is found, Eq. (36) can be written as

$$
F_{\alpha}^{(\text {tail })}=\sum_{l=0}^{\infty}\left(F_{\alpha}^{l}-h_{\alpha}^{l}\right)-D_{\alpha}
$$

where

$$
D_{\alpha} \equiv \lim _{\epsilon \rightarrow 0^{+}} \sum_{l=0}^{\widetilde{\infty}}\left(\delta F_{\alpha}^{l(\epsilon)}-h_{\alpha}^{l}\right)
$$

In principle, a regularization function $h_{\alpha}^{l}$ can be constructed by investigating the asymptotic behavior of $F_{\alpha}^{l}$ as $l \rightarrow \infty$. It is also possible, however, to derive $h_{\alpha}^{l}$ from the large- $l$ asymptotic behavior of $\delta F_{\alpha}^{l(\epsilon)}$ : The latter and $F_{\alpha}^{l}$ must have the same singular behavior at the tilde-limit $l \rightarrow \infty$ (for fixed $\epsilon$ ), as their difference yields a convergent

\footnotetext{
${ }^{5}$ Strictly speaking, the two quantities $F_{\alpha}^{l}$ and $\delta F_{\alpha}^{l(\epsilon)}$ are not well defined without specifying the direction through which the gradient of $G^{l}$ is calculated. This issue is discussed in length later in this section.
} 
tilde-sum over $l$. Obviously, in order to determine $h_{\alpha}^{l}$ (and $D_{\alpha}$ ) from $\delta F_{\alpha}^{l(\epsilon)}$, one merely needs the asymptotic behavior of $\delta F_{\alpha}^{l(\epsilon)}$ in the immediate neighborhood of $\epsilon=0$. This allows one to derive $h_{\alpha}^{l}$ (and $D_{\alpha}$ ) using local analytic methods, as shall be demonstrated in the next section.

First, however, it would be necessary to comment here about a certain indefiniteness involved in the above definitions of the quantities $F_{\alpha}^{l}$ and $\delta F_{\alpha}^{l(\epsilon)}$.

\section{Discontinuity of $F_{\alpha}^{l}$ and $\delta F_{\alpha}^{l(\epsilon)}$}

Whereas the quantity $F_{\alpha}^{l(\epsilon)}$ of Eq. (33) is well defined, the values of the two quantities $F_{\alpha}^{l}$ and $\delta F_{\alpha}^{l(\epsilon)}$ depend on how exactly one evaluates the gradient $G_{, \alpha}^{l}$ at the particle's location. To make this point clear, consider first the $r$-components $F_{r}^{l}$ and $\delta F_{r}^{l(\epsilon)}$. These are calculated according to Eq. (35) from the $r$-derivative of $G^{l}$, reading

$$
G_{, r}^{l}=\frac{L P_{l}(\cos \chi)}{r r^{\prime}}\left\{\left[g_{, r}^{l}-g^{l} / r\right] \Theta\left(v-v^{\prime}\right) \Theta\left(u-u^{\prime}\right)+f^{-1} g^{l}\left[\delta\left(v-v^{\prime}\right) \Theta\left(u-u^{\prime}\right)-\Theta\left(v-v^{\prime}\right) \delta\left(u-u^{\prime}\right)\right]\right\} .
$$

To calculate $F_{r}^{l}$ and $\delta F_{r}^{l(\epsilon)}$ one needs to evaluate this derivative at the self force's evaluation point, $x^{\mu}=x_{0}^{\mu}$, with a source point $x^{\prime \mu}=x_{p}^{\mu}(\tau)$. Now, if the derivative at $x_{0}^{\mu}$ is calculated from $r_{0}^{+}$[namely, by taking the limit $r \rightarrow r_{0}^{+}$of $\left.\frac{G^{l}(r)-G^{l}\left(r_{0}\right)}{r-r_{0}}\right]$, then the $\delta\left(u-u^{\prime}\right)$ term in Eq. (39) will have a nonvanishing contribution to $F_{r}^{l}$ and to $\delta F_{r}^{l(\epsilon)}$ [through the integrals in Eq. (35)], whereas the $\delta\left(v-v^{\prime}\right)$ term will have no contribution - see figure 1. On the other hand, if the derivative is taken from $r^{-}$, it will be the $\delta\left(v-v^{\prime}\right)$ term to contribute, and the $\delta\left(u-u^{\prime}\right)$ term to have no contribution. One can easily verify (as we explicitly do in the following section) that these two different $\delta$ terms yield different contributions to the integrals in Eq. (35). Thus, although each of the quantities $F_{r}^{l}$ and $\delta F_{r}^{l(\epsilon)}$ has well defined values when calculated from either the limit $r \rightarrow r_{0}^{-}$or the limit $r \rightarrow r_{0}^{+}$, these two one-sided values do not coincide. [Note that the quantity $F_{r}^{l(\epsilon)}$ defined in Eq. (33) does not exhibit this kind of discontinuity, as for any finite $\epsilon$ neither of the two $\delta$ terms contribute to this quantity.]
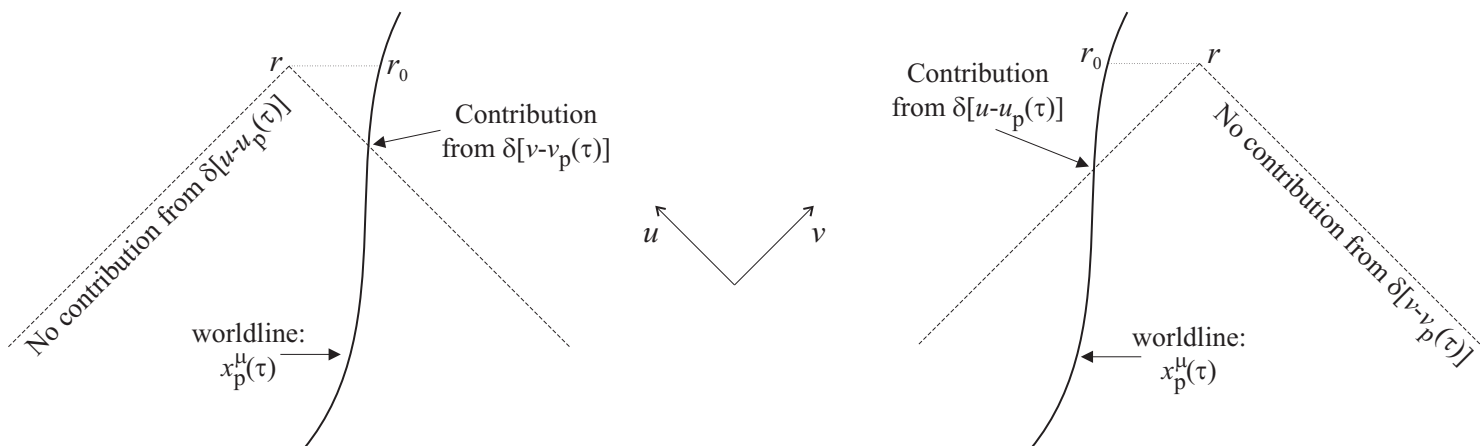

FIG. 1. Discontinuity of $F_{r}^{l}$ and $\delta F_{r}^{l(\epsilon)}$. These quantities are calculated from Eq. 35) by integrating over $\tau$ the Green's function's $r$-derivative at the particle's location, $r=r_{0}$. If this $r$-derivative is calculated from the limit $r \rightarrow r_{0}^{+}$(right figure), then the term in Eq. (39) involving $\delta\left(u-u_{p}\right)$ will contribute to the $\tau$ integration, whereas the $\delta\left(v-v_{p}\right)$ term will have no contribution. The situation is reversed if the $r$-derivative is calculated from the limit $r \rightarrow r_{0}^{-}$(left figure): then, contribution will come only from the $\delta\left(v-v_{p}\right)$ term. Since these two contributions are different, one finds that each of the quantities $F_{r}^{l}$ and $\delta F_{r}^{l(\epsilon)}$ has two different one-sided values (both of which are finite in magnitude).

One can similarly show that the $t$-components $F_{t}^{l}$ and $\delta F_{t}^{l(\epsilon)}$ also exhibit this kind of discontinuity through the particle's location (see the explicit calculation carried out in section $\mathrm{V}$ below). On the other hand, the $\theta$ and $\varphi$ components are obviously continuous through the particle's location, as $G^{l}$ depends on the angular coordinates only through the regular function $P_{l}(\cos \chi)$.

For the sake of definiteness, we shall denote by $F_{r}^{l+}$ and $\delta F_{r}^{l(\epsilon)+}$ the one-sided values arising from the $r \rightarrow r_{0}^{+}$limit, and by $F_{r}^{l-}$ and $\delta F_{r}^{l(\epsilon)-}$ the ones arising from the $r \rightarrow r_{0}^{-}$limit. In addition, the symbols $F_{t}^{l \pm}$ and $\delta F_{t}^{l(\epsilon) \pm}$ will stand for the values derived from the limit $t \rightarrow t_{0}^{\mp}$ if $d r / d t>0$ at the force's evaluation point, or from the limit $t \rightarrow t_{0}^{ \pm}$if $d r / d t<0$ there. (In case $d r / d r=0$ at the force's evaluation point, the two one-sided values of the $t$-component turn out to coincide, as we obtain below.) With this notation we find, for $\alpha=r$ or $t$, 


$$
\delta F_{\alpha}^{l(\epsilon) \pm}=q^{2} \int_{-\epsilon}^{0^{+}} G_{, \alpha}^{l \pm}\left[x_{0}^{\mu} ; x_{p}^{\mu}(\tau)\right] d \tau
$$

(and similarly for $F_{\alpha}^{l \pm}$ ), where

$$
G_{, r}^{l \pm}\left(x^{\mu} ; x^{\prime \mu}\right) \equiv \frac{L P_{l}(\cos \chi)}{r r^{\prime}}\left[g_{, r}^{l}-g^{l} / r \mp f^{-1} g^{l} \delta\left(w_{ \pm}-w_{ \pm}^{\prime}\right)\right],
$$

and

$$
G_{, t}^{l \pm}\left(x^{\mu} ; x^{\prime \mu}\right) \equiv \frac{L P_{l}(\cos \chi)}{r r^{\prime}}\left[g_{, t}^{l}+g^{l} \delta\left(w_{ \pm}-w_{ \pm}^{\prime}\right)\right] .
$$

Here, we have introduced the notation

$$
w_{+} \equiv u \quad \text { and } \quad w_{-} \equiv v
$$

and, likewise, $w_{+}^{\prime} \equiv u^{\prime}$ and $w_{-}^{\prime} \equiv v^{\prime}$.

\section{LOCAL ANALYSIS OF $G^{l}$ FOR LARGE $l$}

The execution of the regularization procedure introduced above involves the construction of the quantities $h_{\alpha}^{l}$ and $D_{\alpha}^{l}$. As we pointed out earlier, this can be done by analyzing $\delta F_{\alpha}^{l(\epsilon)}$, or, more accurately, the quantities $\delta F_{\alpha}^{l(\epsilon) \pm}$ given in Eq. (40). For that goal, we must have sufficient information about the Green's function's $l$-mode $G^{l}$, for large values of $l$, at the immediate vicinity of the self force evaluation point. In this section we use local analysis to obtain analytic approximation for $G^{l}$, up to the accuracy needed for the derivation of $h_{\alpha}^{l}$ and $D_{\alpha}^{l}$.

\section{A. perturbation analysis}

In Sec. III above we have reduced the problem of calculating $G^{l}$ to that of solving a (1+1)-dimensional homogeneous partial DE for the function $g^{l}$, Eq. (27), with the characteristic initial data specified in Eq. (28). Given the function $g^{l}$, the "four-dimensional" Green's function $l$-mode, $G^{l}$, is then constructed from Eq. (30).

To explore the behavior of the function $g^{l}$ for small spacetime intervals and large $l$, we apply the following perturbation analysis. Let us separate the effective potential given in Eq. (23) into two pieces, in the form

$$
V^{l}(r)=L^{2} V_{0}(r)+V_{1}(r),
$$

where

$$
V_{0}(r)=\frac{f(r)}{4 r^{2}}, \quad \text { and } \quad V_{1}(r)=\frac{f(r)}{16 r^{2}}\left[4 r f^{\prime}(r)-1\right]
$$

Let us next expand $V^{l}(r)$ in a Taylor series in the small deviation $r-r_{0}$ about the particle's location $r=r_{0}$. It is convenient to take the small expansion parameter to be $r_{*}-r_{* 0}$, yielding

$$
V^{l}(r)=V^{l}\left(r_{0}\right)+\bar{V}^{l}\left(r_{0}\right)\left(r_{*}-r_{* 0}\right)+\frac{1}{2} \overline{\bar{V}}^{l}\left(r_{0}\right)\left(r_{*}-r_{* 0}\right)^{2}+\cdots,
$$

where an overbar denotes $d / d r^{*}$. Let us also define

$$
\Delta_{r} \equiv 2 V_{00}^{1 / 2} L\left(r_{*}-r_{* 0}\right),
$$

where $V_{00} \equiv V_{0}\left(r_{0}\right)$. We shall refer to a variable of this kind, having the form $L \times$ (small spacetime deviation), as a "neutral" variable. Such "neutral" variables shall play an important role in our analysis, allowing one to properly take into account the delicate interplay between large $l$ and small spacetime deviations. Expressing $r_{*}-r_{* 0}$ in terms of $\Delta_{r} / L$, substituting in the above Taylor expansion, and collecting terms of the same powers in $L$ (with fixed $\Delta_{r}$ ), Eq. (46) takes the form

$$
V^{l}(r)=V_{00}\left[L^{2}+L\left(f_{1} \Delta_{r}\right)+\left(f_{2}+f_{3} \Delta_{r}^{2}\right)\right]+O(1 / L)
$$


where $f_{1}, f_{2}$, and $f_{3}$ are coefficients given by

$$
\begin{aligned}
f_{1} & \equiv \frac{1}{2} V_{00}^{-3 / 2} \bar{V}_{0}=f^{-1 / 2}\left(r f^{\prime}-2 f\right), \\
f_{2} & \equiv V_{00}^{-1} V_{1}=r f^{\prime}-1 / 4, \\
f_{3} & \equiv \frac{1}{8} V_{00}^{-2} \overline{\bar{V}}_{0}=\frac{r^{2}}{2}\left[\left(f^{\prime}\right)^{2} / f+f^{\prime \prime}\right]+3\left(f-r f^{\prime}\right) .
\end{aligned}
$$

(Here, all quantities are evaluated at $r=r_{0}$.)

Defining now the dimensionless "neutral" coordinates

$$
y=V_{00}^{1 / 2} L\left(v-v^{\prime}\right) \quad \text { and } \quad x=V_{00}^{1 / 2} L\left(u-u^{\prime}\right),
$$

Eq. (27) becomes

$$
g_{, y x}^{l}+\left[1+\frac{f_{1} \Delta_{r}}{L}+\frac{f_{2}+f_{3} \Delta_{r}^{2}}{L^{2}}+O\left(1 / L^{3}\right)\right] g^{l}=0 .
$$

We next expand $g^{l}$ in the form

$$
g^{l}=\sum_{k=0}^{\infty} L^{-k} g_{k}\left(\Delta_{r}, \Delta_{r^{\prime}}, z\right)
$$

where the expansion coefficients $g_{k}$ are considered as being dependent of only the "neutral" variables $\Delta_{r}$,

$$
\Delta_{r^{\prime}} \equiv 2 V_{00}^{1 / 2} L\left(r_{*}^{\prime}-r_{* 0}\right)
$$

and

$$
z \equiv 2 \sqrt{x y}=\left(L / r_{0}\right) s .
$$

Here, $s$ is the geodesic distance, to leading order in $r-r^{\prime}$, between the Green's function evaluation and source points (when these two points have the same $\theta$ and $\varphi$ values):

$$
s=\left[f\left(r_{0}\right)\left(v-v^{\prime}\right)\left(u-u^{\prime}\right)\right]^{1 / 2} .
$$

Substituting now the expansion (54) into Eq. (53) and comparing powers of $L$, we obtain a hierarchy of equations for the various functions $g_{k}$, having the form

$$
g_{k, y x}+g_{k}=S_{k}
$$

Here, the source $S_{k}$ is determined for each $k>0$ by the functions $g_{k^{\prime}<k}$ preceding $g_{k}$ in the hierarchy. In the analysis below we shall only need the terms with $k=0,1$, and 2 . For these values of $k$, the source terms are given by

$$
\begin{gathered}
S_{0}=0 \\
S_{1}=-f_{1} \Delta_{r} g_{0}, \\
S_{2}=-f_{1} \Delta_{r} g_{1}-\left(f_{2}+f_{3} \Delta_{r}^{2}\right) g_{0} .
\end{gathered}
$$

Finally, to complete the formulation of a characteristic initial data problem for each of the functions $g_{k}$, we supplement Eq. (58) with the initial conditions

$$
g_{k}\left(v=v^{\prime}\right)=g_{k}\left(u=u^{\prime}\right)=\delta_{k 0},
$$

which conform with the original initial conditions for $g^{l}$, Eq. (28). 


\section{B. Analytic solutions for $k=0,1$, and 2}

The solution to Eq. (58) for $k=0$, subject to the initial conditions, Eq. (60), is given by

$$
g_{0}=J_{0}(z)
$$

where $J_{n}$ are the Bessel functions of the first kind, of order $n$.

To solve for $g_{1}$, we first express the source $S_{1}$ explicitly as a function of $y-x$ and $z$, using Eq. (61) and the relation $\Delta_{r}=y-x+\Delta_{r^{\prime}}$. We find

$$
S_{1}=-f_{1} J_{0}(z)(y-x)-f_{1} \Delta_{r^{\prime}} J_{0}(z)
$$

Then, with the help of Table If we find the solution for $k=1$ [satisfying Eq. (60)] to read

$$
g_{1}=-\frac{1}{4} f_{1} z J_{1}(z)\left(\Delta_{r}+\Delta_{r^{\prime}}\right)
$$

We now use the above solutions for $g_{0}$ and $g_{1}$ to express $S_{2}$ as

$$
S_{2}=\frac{1}{4} f_{1}^{2} z J_{1}(z)\left[(y-x)^{2}+3 \Delta_{r^{\prime}}(y-x)+2 \Delta_{r^{\prime}}^{2}\right]-f_{3} J_{0}(z)\left[(y-x)^{2}+2 \Delta_{r^{\prime}}(y-x)+\Delta_{r^{\prime}}^{2}\right]-f_{2} J_{0}(z) .
$$

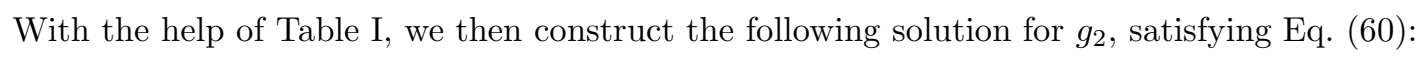

$$
g_{2}=-\frac{1}{6} z J_{1}(z)\left[f_{3}\left(\Delta_{r}^{2}+\Delta_{r} \Delta_{r^{\prime}}+\Delta_{r^{\prime}}^{2}\right)+3 f_{2}\right]+\frac{1}{96} z^{2} J_{2}(z)\left[3 f_{1}^{2}\left(\Delta_{r}+\Delta_{r^{\prime}}\right)^{2}-8 f_{3}\right]+\frac{1}{96} f_{1}^{2} z^{3} J_{3}(z)
$$

\begin{tabular}{|l|l|}
\hline \multicolumn{1}{|c|}{$S(z ; x-y)$} & solution to $g_{, y x}+g=S(z ; x-y)$ \\
\hline$J_{0}(z)$ & $z J_{1}(z) / 2$ \\
$(y-x) J_{0}(z)$ & $(y-x) z J_{1}(z) / 4$ \\
$(y-x)^{2} J_{0}(z)$ & {$\left[z^{2} J_{2}(z)+2(y-x)^{2} z J_{1}(z)\right] / 12$} \\
$z J_{1}(z)$ & $z^{2} J_{2}(z) / 4$ \\
$(y-x) z J_{1}(z)$ & $(y-x) z^{2} J_{2}(z) / 6$ \\
$(y-x)^{2} z J_{1}(z)$ & {$\left[z^{3} J_{3}(z)+3(y-x)^{2} z^{2} J_{2}(z)\right] / 24$} \\
\hline
\end{tabular}

TABLE I. Specific solutions to the inhomogeneous partial DE $g, y x+g=S$ for various source functions of the form $S(z ; x-y)$, where $z=2 \sqrt{x y}$. These solutions can be verified by substitution. In this table, $J_{n}$ are the Bessel functions of the first kind, of order $n$.

\section{C. $G_{, \alpha}^{l \pm}$ expanded in powers of $1 / L$}

We are now in position to write the three leading-order terms in the $1 / L$ expansion of th egradient $G_{, \alpha}^{l \pm}\left[x_{0}^{\mu} ; x_{p}^{\mu}\right]$. To that end we shall need, in view of Eqs. (41) and (42), to evaluate the functions $g_{k}$ derived above, along with their gradients $g_{k, \alpha}$, for $x^{\mu}=x_{0}^{\mu}$ and $x^{\prime \mu}=x_{p}^{\mu}$. For the calculation of $g_{k, \alpha}$ it is convenient to use the auxiliary relations

$$
d\left[z^{n} J_{n}(z)\right] / d z=z^{n} J_{n-1}(z)
$$

[for $n=0$ recall $\left.J_{-1}(z)=-J_{1}(z)\right]$, along with

$$
d z / d r=-f_{0} L(y-x) / z \quad \text { and } \quad d z / d t=f f_{0} L(y+x) / z,
$$

where

$$
f_{0} \equiv\left[r_{0} f^{1 / 2}\left(r_{0}\right)\right]^{-1} .
$$

With the help of these relations we derive from Eqs. 61), 630), and (65) expressions for $g_{k, r}$ and $g_{k, t}$ (where $k=0,1,2$ ). We then set $x^{\mu}=x_{0}^{\mu}$ and $x^{\prime \mu}=x_{p}^{\mu}$ in these expressions, and also in the expressions for the functions $g_{k}$ themselves (noticing the vanishing of $\Delta_{r}$ ). All resulting expressions are then substituted in the formulas for $G_{, r}^{l \pm}$ and $G_{, t}^{l \pm}$, Eqs. 
(41) and (42). In these equations we also make the substitution $\delta\left(w_{ \pm}-w_{ \pm}^{\prime}\right)=2 L V_{00}^{1 / 2} \delta\left(\hat{w}_{ \pm}\right)$where $\hat{w}_{ \pm}$are 'neutral' variables defined by

$$
\hat{w}_{+} \equiv 2 x \quad \text { and } \quad \hat{w}_{-} \equiv 2 y .
$$

Finally, collecting common powers of $L$ we obtain, for $\alpha=r$ or $t$, an expression of the form

$$
G_{, \alpha}^{l \pm}\left[x_{0}^{\mu} ; x_{p}^{\mu}\right]=\frac{P_{l}(\cos \chi)}{r r^{\prime}}\left(\hat{H}_{\alpha}^{(0) \pm} L^{2}+\hat{H}_{\alpha}^{(1)} L+\hat{H}_{\alpha}^{(2)}+\cdots\right)
$$

where the various coefficients $\hat{H}_{\alpha}$ are functions of only the 'neutral' spacetime-interval variables $\widehat{D}_{r^{\prime}}, z, \hat{w}_{ \pm}$, and

$$
\Delta_{t} \equiv 2 V_{00}^{1 / 2} L\left(t_{0}-t_{p}\right)
$$

These coefficient functions are given by

$$
\begin{gathered}
\hat{H}_{r}^{(0) \pm}=f_{0}\left[-\Delta_{r^{\prime}} J_{1}(z) / z \mp J_{0}(z) \delta\left(\hat{w}_{ \pm}\right)\right], \\
\hat{H}_{r}^{(1)}=-\frac{1}{4} f_{0} f_{1}\left[z J_{1}(z)+\Delta_{r^{\prime}}^{2} J_{0}(z)\right]-J_{0}(z) / r_{0}, \\
\hat{H}_{r}^{(2)}=\frac{1}{96} f_{0} \Delta_{r^{\prime}}\left[7 f_{1}^{2} z^{2} J_{2}(z)+3 z J_{1}(z)\left(f_{1}^{2} \Delta_{r^{\prime}}^{2}-8 f_{3}\right)-16 J_{0}(z)\left(f_{3} \Delta_{r^{\prime}}^{2}+3 f_{2}\right)\right]+\frac{1}{4} f_{1} \Delta_{r^{\prime}} z J_{1}(z) / r_{0},
\end{gathered}
$$

and

$$
\begin{gathered}
\hat{H}_{t}^{(0) \pm}=f f_{0}\left[-\Delta_{t} J_{1}(z) / z+J_{0}(z) \delta\left(\hat{w}_{ \pm}\right)\right] \\
\hat{H}_{t}^{(1)}=-\frac{1}{4} f f_{0} f_{1} \Delta_{r^{\prime}} \Delta_{t} J_{0}(z) \\
\hat{H}_{t}^{(2)}=\frac{1}{96} f f_{0} \Delta_{t}\left[f_{1}^{2} z^{2} J_{2}(z)+z J_{1}(z)\left(3 f_{1}^{2} \Delta_{r^{\prime}}^{2}-8 f_{3}\right)-16 J_{0}(z)\left(f_{3} \Delta_{r^{\prime}}^{2}+3 f_{2}\right)\right]
\end{gathered}
$$

(where the function $f$ is to be evaluated at $r=r_{0}$ ). In the above expressions for $\hat{H}_{r}^{(1),(2)}$ and $\hat{H}_{t}^{(1),(2)}$ we have omitted terms of the form $\propto z^{k} J_{n}(z) \delta\left(\hat{w}_{ \pm}\right)$, with $k$ being a positive integer, as such terms would yield vanishing contributions to $\delta F_{\alpha}^{l(\epsilon) \pm}$ when integrated over $\tau$ in Eq. (40).

The quantities $\delta F_{\alpha}^{l(\epsilon) \pm}$ can now be constructed, in principle, for any given worldline, by inserting Eq. (70) into Eq. (40) and carrying out the integration over $\tau$. In practice, to integrate over $\tau$, one should proceed as follows: Recalling that $\tau$ is a small quantity (we have $|\tau| \leq \epsilon$ ), one first expands in powers of $\tau$ all $\tau$-dependent quantities in the integrand of Eq. (40). (At that point, the details of the specific trajectory under consideration enter the calculation in an explicit manner; specifically, the power expansion coefficients turn out to depend on the values of $u^{\alpha}$, $\dot{u}^{\alpha}$, and $\ddot{u}^{\alpha}$ at the force's evaluation point.) Then, since we are interested in extracting the large $l$ (large $L$ ) behavior of $\delta F_{\alpha}^{l(\epsilon) \pm}$, we introduce the "neutral" dimensionless proper time variable, defined by

$$
\lambda \equiv-\left(L / r_{0}\right) \tau,
$$

and replace $\tau$ by $-r_{0}(\lambda / L)$. The integrand then takes the form of a power series in $1 / L$, with $\lambda$-dependent coefficients. Transforming finally from integration over $\tau$ to integration over $\lambda$, one obtains an expression for $\delta F_{\alpha}^{l(\epsilon) \pm}$ in the form of a power series in $1 / L$, as desired. In the rest of this paper we carry out the above calculation in full detail (and derive $h_{\alpha}^{l}$ and $D_{\alpha}$ ) for the case of a purely radial trajectory.

\footnotetext{
${ }^{6}$ We hereafter use the symbols $\Delta_{r^{\prime}}, z$, and $\hat{w}_{ \pm}$to represent the values of these variables for $x^{\mu}=x_{0}^{\mu}$ and $x^{\prime \mu}=x_{p}^{\mu}$.
} 


\section{FORM OF THE REGULARIZATION FUNCTION $h^{l}$ : THE CASE OF RADIAL MOTION}

When considering radial trajectories (namely, ones along which $d \theta=d \varphi=0$ ) one has $P_{l}(\cos \chi) \equiv 1$. Consequently, the Green's function given in Eq. (30) becomes $\theta, \varphi$-independent, resulting in the vanishing of both angular components of the self force (as should be expected, of course, by virtue of the background being spherically-symmetric). In the following we discuss the $r$ and $t$ components of the self-force.

To carry out the integration in Eq. (40) we first expand each $\tau$-dependent quantity in the integrand in powers of $1 / L$, with $\lambda$ held fixed. The $\tau$-dependent quantities to be expanded are $\Delta_{r^{\prime}}, \Delta_{t}, 1 / r^{\prime}, z$, and the various Bessel functions appearing in Eqs. (72) and (73).

By expanding $\Delta_{r^{\prime}}$ in a Taylor series in $\tau$ about $r=r_{0}$, and transforming to the variable $\lambda$, we obtain the expansion

$$
\begin{aligned}
\Delta_{r^{\prime}}= & \dot{\Delta}_{r^{\prime}} \tau+\frac{1}{2} \ddot{\Delta}_{r^{\prime}} \tau^{2}+\frac{1}{6} \dddot{\Delta}_{r^{\prime}} \tau^{3}+\cdots= \\
& f^{1 / 2}\left[-\dot{r}_{*} \lambda+\frac{1}{2} \ddot{r}_{*} \lambda^{2}\left(r_{0} / L\right)-\frac{1}{6} \dddot{r}_{*} \lambda^{3}\left(r_{0} / L\right)^{2}\right]+O(1 / L)^{3},
\end{aligned}
$$

where

$$
\begin{aligned}
\dot{r}_{*} & =f^{-1} \dot{r} \\
\ddot{r}_{*} & =f^{-2}\left(f \ddot{r}-f^{\prime} \dot{r}^{2}\right), \\
\dddot{r}_{*} & =f^{-3}\left[\left(2 f^{\prime 2}-f^{\prime \prime} f\right) \dot{r}^{3}-3 f^{\prime} f \dot{r} \ddot{r}+f^{2} \dddot{r}\right],
\end{aligned}
$$

and where all quantities (except $\lambda$ ) are evaluated at $r=r_{0}(\tau=0)$. In a similar manner we obtain for $\Delta_{t}$,

$$
\Delta_{t}=f^{1 / 2}\left[\dot{t} \lambda-\frac{1}{2} \ddot{t} \lambda^{2}\left(r_{0} / L\right)+\frac{1}{6} \dddot{t} \lambda^{3}\left(r_{0} / L\right)^{2}\right]+O(1 / L)^{3}
$$

and for $1 / r^{\prime}$

$$
\left.\frac{1}{r^{\prime}}=\frac{1}{r_{0}}\left[1+\dot{r}(\lambda / L)+\frac{1}{2}\left(2 \dot{r}^{2}-r_{0} \ddot{r}\right)(\lambda / L)^{2}\right)\right]+O(1 / L)^{3} .
$$

Next, recalling $z=\left(L / r_{0}\right) s$, we obtain

$$
z=-\dot{s} \lambda+\frac{1}{2} \ddot{s} \lambda^{2}\left(r_{0} / L\right)-\frac{1}{6} \dddot{s} \lambda^{3}\left(r_{0} / L\right)^{2}+O(1 / L)^{3} .
$$

To calculate the $\tau$-derivatives of $s$ (which are understood here to be evaluated at $\tau=0$ ), we make use of the normalization relation $\dot{v} \dot{u}=1 / f$, and of the relations derived from it by successively differentiating its both sides with respect to $\tau: \ddot{v} \dot{u}+\dot{v} \ddot{u}=(1 / f)^{\prime} \dot{r}$, and $\dddot{v} \dot{u}+2 \ddot{v} \ddot{u}+\dot{v} \dddot{u}=(1 / f)^{\prime \prime} \dot{r}^{2}+(1 / f)^{\prime} \ddot{r}$. Using these relations we find

$$
\begin{aligned}
\dot{s}(\tau=0) & =-1 \\
\ddot{s}(\tau=0) & =\frac{1}{2}\left(f^{\prime} / f\right) \dot{r} \\
\dddot{s}(\tau=0) & =\frac{1}{16 f^{2}}\left[\left(8 f^{\prime \prime} f-13{f^{\prime}}^{2}\right) \dot{r}^{2}+8 f^{\prime} f \ddot{r}\right]+\frac{1}{4} f \ddot{v} \ddot{u}
\end{aligned}
$$

(note that whereas $\tau$ is non-positive throughout the integration domain, $z$ and $s$ are, by definition, non-negative).

Finally, we need to similarly expand the various Bessel functions appearing in the integrand of Eq. (40). Using Eqs. (79) and (80) we find for any $n \geq 0$,

$$
J_{n}(z)=J_{n}(\lambda)+\frac{1}{2}\left(r_{0} / L\right) \ddot{s} \lambda^{2} J_{n}^{\prime}(\lambda)+\left(r_{0} / L\right)^{2}\left(\frac{1}{8} \ddot{s}^{2} \lambda^{4} J_{n}^{\prime \prime}(\lambda)-\frac{1}{6} \dddot{s} \lambda^{3} J_{n}^{\prime}(\lambda)\right)+O\left(1 / L^{3}\right),
$$

where a prime denotes $d / d \lambda$. Using this general form together with Eq. (66), we obtain the following expansions, needed for our analysis: 


$$
\begin{aligned}
J_{0}(z)= & J_{0}(\lambda)-\frac{1}{2}\left(r_{0} / L\right) \ddot{s} \lambda^{2} J_{1}(\lambda)+O\left(1 / L^{2}\right) \\
J_{1}(z)= & J_{1}(\lambda)+\frac{1}{2}\left(r_{0} / L\right) \ddot{s}\left(\lambda^{2} J_{0}(\lambda)-\lambda J_{1}(\lambda)\right) \\
& +\left(r_{0} / L\right)^{2}\left[\frac{1}{8} \ddot{s}^{2}\left(\lambda^{3} J_{2}(\lambda)-\lambda^{4} J_{1}(\lambda)\right)-\frac{1}{6} \dddot{s}\left(\lambda^{3} J_{0}(\lambda)-\lambda^{2} J_{1}(\lambda)\right)\right]+O\left(1 / L^{3}\right) .
\end{aligned}
$$

We now substitute the above expansions for $\Delta_{r^{\prime}}, \Delta_{t}, z$, and the Bessel functions in Eqs. (72)-(73). We also substitute for the delta functions in Eqs. (72a) and (73a)

$$
\delta\left(\hat{w}_{ \pm}\right)=\frac{\delta(\lambda)}{\left|d \hat{w}_{ \pm} / d \lambda\right|}=\frac{\delta(\lambda)}{f^{1 / 2} \dot{w}_{ \pm}}=f^{1 / 2} \dot{w}_{\mp} \delta(\lambda),
$$

where the last equality is due to the normalization of the four-velocity. We thereby obtain expressions for the various functions $\hat{H}_{\alpha}^{(n=0,1,2)}$, each expanded in powers of $1 / L$ up to order $O\left(L^{n-2}\right)$ (with $\lambda$-dependent coefficients). Substitution of these expressions [and of the expansion for $1 / r^{\prime}$, Eq. (78)] into Eq. (70) finally yields the desired expression for the Green's function's gradient, as a power series in $1 / L$ (with $\lambda$ held fixed). We find (for $\alpha=r, t$ )

$$
G_{, \alpha}^{l \pm}=H_{\alpha}^{(0) \pm} L^{2}+H_{\alpha}^{(1)} L+H_{\alpha}^{(2)}+O(1 / L),
$$

where the various coefficients $H_{\alpha}^{(n)}$ are functions of $\lambda$ along the worldline, given by

$$
\begin{gathered}
H_{r}^{(0) \pm}=\frac{1}{r_{0}^{3}}\left[\dot{r}_{*} J_{1}(\lambda) \mp \dot{w}_{\mp} J_{0}(\lambda) \delta(\lambda)\right] \\
H_{r}^{(1)}=-\frac{1}{4 r_{0}^{3}}\left[\lambda J_{1}(\lambda)\left(f_{1} / f^{1 / 2}-4 \dot{r} \dot{r}_{*}+4 r_{0} \ddot{s} \dot{r}_{*}+2 r_{0} \ddot{r}_{*}\right)\right. \\
\left.+\lambda^{2} J_{0}(\lambda)\left(f^{1 / 2} f_{1} \dot{r}_{*}^{2}-2 r_{0} \ddot{s}_{*}\right)+4 J_{0}(\lambda)\right] \\
H_{r}^{(2)=-} \frac{1}{96 r_{0}^{3}}\left[48 \lambda J_{0}(\lambda)\left(2 \dot{r}-f_{2} \dot{r}_{*}\right)\right. \\
+4 \lambda^{3} J_{0}(\lambda)\left(3 f_{1} r_{0} \ddot{s} / f^{1 / 2}+6 r_{0}^{2} \ddot{s}^{2} \dot{r}_{*}+4 r_{0}^{2} \dddot{s}_{*}-4 f f_{3} \dot{r}_{*}^{3}+6 r_{0}^{2} \ddot{s}_{*}-6 f^{1 / 2} f_{1} r_{0} \dot{r}_{*} \ddot{r}_{*}\right. \\
\left.-12 \dot{r} r_{0} \ddot{s} \dot{r}_{*}+6 f^{1 / 2} f_{1} \dot{r} \dot{r}_{*}^{2}\right) \\
+8 \lambda^{2} J_{1}(\lambda)\left(3 f_{1} \dot{r} / f^{1 / 2}-6 r_{0} \ddot{s}-3 f_{3} \dot{r}_{*}-12 \dot{r}^{2} \dot{r}_{*}+6 r_{0} \ddot{r} \dot{r}_{*}+12 r_{0} \dot{r} \ddot{s} \dot{r}_{*}\right. \\
\left.-6 r_{0}^{2} \ddot{s}^{2} \dot{r}_{*}-4 r_{0}^{2} \dddot{s} \dot{r}_{*}+6 r_{0} \dot{r} \ddot{r}_{*}-6 r_{0}^{2} \dddot{s} \ddot{r}_{*}-2 r_{0}^{2} \dddot{r}_{*}+3 f^{1 / 2} f_{1} \dot{r}_{*}\right) \\
+3 \lambda^{4} J_{1}(\lambda)\left(4 r_{0}^{2} \ddot{s}^{2} \dot{r}_{*}+f f_{1}^{2} \dot{r}_{*}^{3}-4 f^{1 / 2} f_{1} r_{0} \ddot{s}_{*}^{2}\right) \\
\left.+\lambda^{3} J_{2}(\lambda)\left(7 f_{1}^{2} \dot{r}_{*}-12 r_{0}^{2} \ddot{s}^{2} \dot{r}_{*}\right)\right]
\end{gathered}
$$

and

$$
\begin{gathered}
H_{t}^{(0) \pm}=\frac{f}{r_{0}^{3}}\left[J_{0}(\lambda) \dot{w}_{\mp} \delta(\lambda)-J_{1}(\lambda) \dot{t}\right], \\
H_{t}^{(1)}=\frac{f}{4 r_{0}^{3}}\left\{\lambda J_{1}(\lambda)\left[-4 \dot{r} \dot{t}+2 r_{0}(2 \ddot{s} \dot{t}+\ddot{t})\right]+\lambda^{2} J_{0}(\lambda)\left(-2 r_{0} \ddot{s} \dot{t}+f^{1 / 2} f_{1} \dot{r}_{*} \dot{t}\right)\right\}, \\
H_{t}^{(2)}=-\frac{f}{96 r_{0}^{3}}\left\{48 \lambda J_{0}(\lambda) f_{2} \dot{t}\right. \\
-4 \lambda^{3} J_{0}(\lambda)\left[r_{0}^{2}\left(6 \ddot{s}^{2} \dot{t}+4 \dddot{s} \ddot{t}+6 \ddot{s} \ddot{t}\right)-3 r_{0}\left(4 \dot{r} \ddot{s} \dot{t}+f^{1 / 2} f_{1} \ddot{t} \dot{r}_{*}+f^{1 / 2} f_{1} \dot{t} \ddot{r}_{*}\right)+6 f^{1 / 2} f_{1} \dot{r} \dot{t} \dot{r}_{*}-4 f f_{3} \dot{t} \dot{r}_{*}^{2}\right] \\
+8 \lambda^{2} J_{1}(\lambda)\left(f_{3} \dot{t}+12 \dot{r}^{2} \dot{t}-6 r_{0} \ddot{r} \ddot{t}-12 r_{0} \dot{r} \ddot{s} \dot{t}+6 r_{0}^{2} \ddot{s}^{2} \dot{t}+4 r_{0}^{2} \dddot{s} \dot{t}-6 r_{0} \dot{r} \ddot{t}+6 r_{0}^{2} \ddot{s} \ddot{t}+2 r_{0}^{2} \dddot{t}\right) \\
+3 \lambda^{4} J_{1}(\lambda)\left(-4 r_{0}^{2} \ddot{s}^{2} \dot{t}+4 f^{1 / 2} f_{1} r_{0} \ddot{s} \ddot{t} \dot{r}_{*}-f f_{1}^{2} \dot{t} \dot{r}_{*}^{2}\right) \\
\left.+\lambda^{3} J_{2}(\lambda)\left(-f_{1}^{2} \dot{t}+12 r_{0}^{2} \ddot{s}^{2} \dot{t}\right)\right\} .
\end{gathered}
$$


Changing the integration variable in Eq. (40) from $\tau$ to $\lambda$, we now have

$$
\delta F_{\alpha}^{l(\epsilon) \pm}=q^{2} r_{0} \int_{0}^{L \epsilon / r_{0}}\left[L H_{\alpha}^{(0) \pm}+H_{\alpha}^{(1)}+H_{\alpha}^{(2)} / L+O\left(L^{-2}\right)\right] d \lambda
$$

The desired regularization function $h_{\alpha}^{l}$ is to be constructed such as to extract the large $l$ singular behavior of $\delta F_{\alpha}^{l(\epsilon) \pm}$ (while maintaining the simplest form possible). By virtue of Eq. (94) we take this function to be

$$
h_{\alpha}^{l \pm}=L A_{\alpha}^{ \pm}+B_{\alpha}+C_{\alpha} / L
$$

where

$$
\begin{gathered}
A_{\alpha}^{ \pm} \equiv \widetilde{\lim }_{l \rightarrow \infty}\left(L^{-1} \delta F_{\alpha}^{l(\epsilon) \pm}\right)=q^{2} r_{0} \int_{0}^{\widetilde{\infty}} H_{\alpha}^{(0) \pm}(\lambda) d \lambda, \\
B_{\alpha} \equiv \widetilde{\lim _{l \rightarrow \infty}}\left(\delta F_{\alpha}^{l(\epsilon) \pm}-L A_{\alpha}^{ \pm}\right)=q^{2} r_{0} \int_{0}^{\widetilde{\infty}} H_{\alpha}^{(1)}(\lambda) d \lambda, \\
C_{\alpha} \equiv \widetilde{\lim }_{l \rightarrow \infty} L\left(\delta F_{\alpha}^{l(\epsilon) \pm}-L A_{\alpha}^{ \pm}-B_{\alpha}\right)=q^{2} r_{0} \int_{0}^{\widetilde{\infty}} H_{\alpha}^{(2)}(\lambda) d \lambda,
\end{gathered}
$$

with $\int^{\widetilde{\infty}}() d \lambda$ standing for $\widetilde{\lim }_{x \rightarrow \infty} \int^{x}() d \lambda$. 7 That the second equality in each of Eqs. (96b) and (96c) is valid, and that the above choice of function $h_{\alpha}^{l \pm}$ indeed satisfies the requirement that the tilde-sum $\sum_{l=0}^{\widetilde{\infty}}\left(\delta F_{\alpha}^{l(\epsilon) \pm}-h_{\alpha}^{l \pm}\right)$ would converge, will be shown in the next section, where we explicitly calculate the parameters $A_{\alpha}, B_{\alpha}$, and $C_{\alpha}$. The reason for using the tilde-limit instead of the standard limit in the definitions of these parameters will also become clear then. As to the parameter $D_{\alpha}$, substituting now Eqs. (94) and (95) into Eq. (38), one obtains

$$
D_{\alpha}=-q^{2} r_{0} \lim _{\epsilon \rightarrow 0} \sum_{l=0}^{\widetilde{\infty}} \int_{L \epsilon / r_{0}}^{\widetilde{\infty}}\left[L H_{\alpha}^{(0) \pm}+H_{\alpha}^{(1)}+H_{\alpha}^{(2)} / L\right] d \lambda .
$$

In conclusion, we find the tail part of the self force to be given by

$$
F_{\alpha}^{(\text {tail })}=\sum_{l=0}^{\infty}\left(F_{\alpha}^{l \pm}-A_{\alpha}^{ \pm} L-B_{\alpha}-C_{\alpha} / L\right)-D_{\alpha},
$$

where, from the above construction of $h_{\alpha}^{l}$, it follows that the sum over $l$ converges at least as $\sim 1 / l$. The implementation of our regularization scheme thus amounts to analytically determining the regularization parameters $A_{\alpha}^{ \pm}, B_{\alpha}, C_{\alpha}$, and $D_{\alpha}$, using Eqs. (96) and (97). For the calculation of the tail term, one may use either $F_{\alpha}^{l+}$ (with $A_{\alpha}^{+}$) or $F_{\alpha}^{l-}$ (with $\left.A_{\alpha}^{-}\right)$. Of course, one may also use any combination of these two one-sided quantities (e.g., their average). It should be emphasized here that the final result of the calculation, namely the tail term $F_{\alpha}^{\text {(tail) }}$ (having a well defined value at the evaluation point), should be the same regardless of whether it is derived from one of the one-sided limits, or from the other, or, say, from their average.

We finally point out that, although Eq. (98) has been developed here for radial motion, an expression of this form is also valid for any other trajectory [29]. The details of the specific trajectory under consideration would only affect the values of the various regularization parameters.

\footnotetext{
${ }^{7}$ Here we extend the definition of the tilde-limit, given in App. A, from discrete functions with index $l$ to continuous functions of $\lambda$. In an analogous manner, the tilde-limit of a function $f(\lambda)$ as $\lambda \rightarrow \infty$ would be defined through the subtraction of a finite sum of functions of the form $B^{(j)}(\lambda)=a_{j} \lambda^{b_{j}} \cos \left(\alpha_{j} \lambda+\beta_{j}\right.$ ) (with $\alpha_{j} \neq 0$ for all $j$ ). It is simple to verify that the tilde-limit of a function, when existing, is single-valued.

${ }^{8}$ We assume here that the contribution associated with the $O\left(L^{-2}\right)$ term in Eq. (94) vanishes upon taking the limit $\epsilon \rightarrow 0$, as this contribution is of order $O(\epsilon)$ (this becomes clear from the calculation of $D_{\alpha}$ in the next section). However, a problem may occur if this term fails to yield a finite contribution when integrated over $\lambda$. Here we do not further investigate the behavior of the $O\left(L^{-2}\right)$ term, and just assume that the above potential problem is not realized.
} 


\section{DERIVATION OF THE REGULARIZATION PARAMETERS FOR RADIAL MOTION}

To carry out the calculation of the regularization parameters in this section, we shall need the following integrals, the derivation of which will be described in Appendix $\mathbb{B}$. For $k, n \in \mathbb{N}$ we have

$$
\int_{0}^{\widetilde{\infty}} \lambda^{k} J_{n}(\lambda) d \lambda= \begin{cases}(n+k-1) ! ! /(n-k-1) ! !, & 0 \leq k \leq n \\ (-1)^{(k-n) / 2}(k+n-1) ! !(k-n-1) ! !, & \text { even } k-n>0 \\ 0, & \text { odd } k-n>0\end{cases}
$$

[in applying this formula for $k=n$, recall $(-1) ! !=1$ ]. If the difference $k-n$ is a positive odd integer, then we also have

$$
\int_{0}^{\widetilde{\infty}} \lambda^{k} J_{n}(\lambda) \ln \lambda d \lambda=(-1)^{(k-n+1) / 2}(k+n-1) ! !(k-n-1) ! !
$$

\section{A. Derivation of $A_{\alpha}^{ \pm}$}

Substituting Eqs. (38) and (91) into Eq. (96a) and carrying out the tilde-integration [with the help of Eq. (99)] we find, recalling $J_{0}(0)=1$ and $J_{0}(\infty)=0$,

$$
\begin{aligned}
& A_{r}^{ \pm}=\frac{q^{2}}{r_{0}^{2}}\left(\dot{r}_{*} \mp \dot{w}_{\mp}\right)=\mp \frac{q^{2}}{r_{0}^{2}} \dot{t}, \\
& A_{t}^{ \pm}=f \frac{q^{2}}{r_{0}^{2}}\left(-\dot{t}+\dot{w}_{\mp}\right)= \pm \frac{q^{2}}{r_{0}^{2}} \dot{r} .
\end{aligned}
$$

Note that the two one-sided values of $A_{\alpha}$ are, in general, not the same. Consequently, as argued above, the function $h_{\alpha}^{l}$ (and also $\delta F_{\alpha}^{l(\epsilon)}$ ) exhibits two different one-sided values. We note that the averaged value of the parameter $A_{\alpha}$, to be denoted by $\bar{A}_{\alpha}$, is found to vanish:

$$
\bar{A}_{\alpha} \equiv \frac{1}{2}\left(A_{\alpha}^{+}+A_{\alpha}^{-}\right)=0
$$

This vanishing of $\bar{A}_{\alpha}$ seems to occur for all trajectories of a scalar particle, not only the radial ones considered here [29.

\section{B. Derivation of $B_{\alpha}$}

By the definition of $B_{\alpha}$ in Eq. (96b) we have, after substituting for $A_{\alpha}^{ \pm}$from Eq. (96a),

$$
B_{\alpha}=-q^{2} r_{0} \widetilde{\lim }_{l \rightarrow \infty}\left[L \int_{L \epsilon / r_{0}}^{\widetilde{\infty}} H_{\alpha}^{(0) \pm}(\lambda) d \lambda\right]+q^{2} r_{0} \int_{0}^{\widetilde{\infty}} H_{\alpha}^{(1)}(\lambda) d \lambda .
$$

The first term here cancels out upon taking the tilde-limit $l \rightarrow \infty$ : At large $l$ (and fixed $\epsilon$ ), each of the components $H_{\alpha}^{(0) \pm}$ behaves as $\propto l^{-1 / 2}$ times oscillations with respect to $l$ [these components are linear combinations of Bessel functions, the asymptotic form of which is described in Eq. (B6)]. To leading order in 1/l, this is also the form of the integral over $H_{\alpha}^{(0) \pm}$ in Eq. (103) (which is carried out over asymptotically large values of $\lambda$ ). Hence, the expression in the squared brackets is found to diverge as $\propto l^{1 / 2}$ times oscillations. When taking the tilde-limit, this divergent piece is removed, with the remaining part dying off at large $l$ as $\propto l^{-1 / 2}$ (times oscillations). Therefore, no contribution arises from the first term in Eq. (103), and the second equality of Eq. (96b) is shown to be valid.

To calculate the parameter $B_{\alpha}$, we now substitute for $H_{\alpha}^{(1)}$ from Eqs. (89) and (92) (for the $r$ and $t$ components, respectively). The calculation involves tilde-integrating over terms of the form $\propto \lambda J_{1}(\lambda), \propto \lambda^{2} J_{0}(\lambda)$, and $\propto J_{0}(\lambda)$. Reading the values of the these integrals from Eq. (99), and substituting for $f_{1}, \dot{r}_{*}, \ddot{r}_{*}$, and $\ddot{s}$ [using Eqs. (49), (76), and (81)], we obtain 


$$
B_{r}=-\frac{q^{2}}{2 r_{0}^{2}} f^{-1}\left(f+r_{0} f^{\prime} / 2-\dot{r}^{2}+r_{0} \ddot{r}\right)
$$

and

$$
B_{t}=\frac{q^{2}}{2 r_{0}^{2}}\left[f r_{0} \ddot{t}+\dot{t} \dot{r}\left(r_{0} f^{\prime}-f\right)\right]
$$

Recalling $a^{r}=\ddot{r}+f^{\prime} / 2$ and $a^{t}=\ddot{t}+f^{\prime} \dot{r}_{*} \dot{t}$ in the case of radial motion considered here, we may express this result in a more compact form as

$$
B_{\alpha}=-\frac{q^{2}}{2 r_{0}^{2}}\left(\delta_{\alpha}^{r}+r_{0} a_{\alpha}-\dot{r} u_{\alpha}\right)
$$

\section{Derivation of $C_{\alpha}$}

By its definition in Eq. (960), we have for the parameter $C_{\alpha}$, after substituting for $A_{\alpha}$ and $B_{\alpha}$,

$$
C_{\alpha}=-q^{2} r_{0} \widetilde{\lim }_{l \rightarrow \infty}\left[\int_{L \epsilon / r_{0}}^{\widetilde{\infty}}\left(L H_{\alpha}^{(0)}(\lambda)+H_{\alpha}^{(1)}(\lambda)\right) d \lambda\right]+q^{2} r_{0} \int_{0}^{\widetilde{\infty}} H_{\alpha}^{(2)}(\lambda) d \lambda .
$$

Again, there is a residual part left from the calculation of $A_{\alpha}$ and $B_{\alpha}$, which involves integration over asymptotically large values of $\lambda$. This part can again be shown to vanish as the tilde-limit $l \rightarrow \infty$ is taken, resulting in that only the second integral in Eq. (107) survives.

To calculate $C_{\alpha}$, we thus use the second equality of Eq. (96c), in which we substitute for $H_{\alpha}^{(2)}$ from Eqs. (90) and (93) (for the $r$ and $t$ components, respectively). One then has to evaluates the tilde-integral of a sum of various terms of the form $\propto \lambda^{k} J_{n}(\lambda)$, all of which have $k>n$ and odd $k-n$. According to Eq. (99), all such integrals vanish. Thus, we find

$$
C_{\alpha}=0
$$

The vanishing of the parameter $C_{\alpha}$ seems to be a universal feature of our scheme, regardless of the specific trajectory under consideration 29]. As we also find below, this vanishing constitutes a necessary condition for the self-consistency of the whole regularization scheme.

\section{Derivation of $D_{\alpha}$}

To calculate the parameter $D_{\alpha}$ we write Eq. (97) in the form

$$
D_{\alpha}=D_{\alpha}^{(0)}+D_{\alpha}^{(1)}+D_{\alpha}^{(2)}
$$

where

$$
D_{\alpha}^{(n)} \equiv-q^{2} r_{0} \lim _{\epsilon \rightarrow 0} \sum_{l=0}^{\widetilde{\infty}} \int_{L \epsilon / r_{0}}^{\widetilde{\infty}} L^{1-n} H_{\alpha}^{(n) \pm}(\lambda) d \lambda
$$

In calculating the above three pieces of $D_{\alpha}$, we shall transform from summation over $l$ to integration over a continuous variable. For this transformation we will make use of the relation

$$
\sum_{l=0}^{\infty}\left(\epsilon / r_{0}\right) K\left(L \epsilon / r_{0}\right)=\int_{0}^{\infty}\left[K(x)-\frac{1}{24}\left(\epsilon / r_{0}\right)^{2} K^{\prime \prime}(x)\right] d x+O\left(\epsilon^{3}\right)
$$

where $K(x)$ is any (sufficiently regular) integrable real function, and $x$ is an integration variable. Here, the $O\left(\epsilon^{0}\right)$ term on the right-hand side (RHS) is the standard ("Riemann type") integral, which is obtained, by definition, when the $\epsilon \rightarrow 0$ limit of the left-hand side is taken. We also indicated here the $O\left(\epsilon^{2}\right)$ correction to the integral, which we 
shall have to take into account in the calculation below (it is straightforward to verify the form of this correction term using standard calculus). Obviously, Eq. (111) also holds for the tilde-sum $\sum_{l=0}^{\widetilde{\infty}}$, where on the RHS we use the tilde-integral $\int_{0}^{\widetilde{\infty}}$.

Beginning with the calculation of $D_{\alpha}^{(0)}$, we write Eq. (110) for $n=0$ as

$$
D_{\alpha}^{(0)}=-q^{2} r_{0} \lim _{\epsilon \rightarrow 0}\left[\left(r_{0} / \epsilon\right)^{2} \sum_{l=0}^{\widetilde{\infty}}\left(\epsilon / r_{0}\right)\left(\left(L \epsilon / r_{0}\right) \int_{L \epsilon / r_{0}}^{\widetilde{\infty}} H_{\alpha}^{(0) \pm}(\lambda) d \lambda\right)\right] .
$$

Comparing the form of the sum in this expression to the left-hand side of Eq. (111), we find

$$
D_{\alpha}^{(0)}=-q^{2} r_{0} \lim _{\epsilon \rightarrow 0}\left[\left(r_{0} / \epsilon\right)^{2} \int_{0}^{\widetilde{\infty}} d x\left(K_{\alpha}^{(0)}(x)-\frac{1}{24}\left(\epsilon / r_{0}\right)^{2}\left[K_{\alpha}^{(0)}(x)\right]^{\prime \prime}\right)\right],
$$

where

$$
K_{\alpha}^{(0)}(x) \equiv x \int_{x}^{\widetilde{\infty}} H_{\alpha}^{(0) \pm}(\lambda) d \lambda .
$$

Note here how the contribution to $D_{\alpha}^{(0)}$ due to the $O\left(\epsilon^{3}\right)$ term appearing in Eq. (111) vanishes upon taking the limit $\epsilon \rightarrow 0$. We recall that each of the two components $H_{r}^{(0) \pm}$ and $H_{t}^{(0) \pm}$, given explicitly in Eqs. (88) and (91), contains two terms: one proportional to $\delta(\lambda)$ and the other to $J_{1}(\lambda)$. The $\propto \delta(\lambda)$ term has no contribution to $K_{\alpha}^{(0)}$, resulting in that both two one-sided values $H_{\alpha}^{(0)+}$ and $H_{\alpha}^{(0)-}$ yield the same function $K_{\alpha}^{(0)}$ (for that reason, no \pm sign has been assigned to this quantity). There is an apparent danger of divergence coming from the $O\left(\epsilon^{-2}\right)$ term in Eq. 113 . Such a divergence is avoided, however, as we have

$$
\int_{0}^{\widetilde{\infty}} K_{\alpha}^{(0)}(x) d x=\frac{u_{\alpha}}{r_{0}^{3}} \int_{0}^{\widetilde{\infty}} d x x \int_{x}^{\widetilde{\infty}} J_{1}(\lambda) d \lambda=\frac{u_{\alpha}}{2 r_{0}^{3}} \int_{0}^{\widetilde{\infty}} x^{2} J_{1}(x) d x=0,
$$

where in the second equality we integrated by parts with respect to $x$, and where the vanishing of the last integral is implied by Eq. (99). Thus, the $O\left(\epsilon^{-2}\right)$ term in Eq. (113) vanishes, and the $\epsilon \rightarrow 0$ limit in this equation turns out well defined. The remaining $O\left(\epsilon^{0}\right)$ contribution reads

$$
D_{\alpha}^{(0)}=\frac{q^{2} r_{0}}{24} \int_{0}^{\widetilde{\infty}}\left[K_{\alpha}^{(0)}(x)\right]^{\prime \prime} d x=-\frac{q^{2} r_{0}}{24} \int_{0}^{\widetilde{\infty}}\left\{H_{\alpha}^{(0) \pm}(x)+\left[x H_{\alpha}^{(0) \pm}(x)\right]^{\prime}\right\} d x=-\frac{q^{2} r_{0}}{24} \int_{0}^{\widetilde{\infty}} H_{\alpha}^{(0) \pm}(x) d x
$$

as the surface term vanishes. Substituting for $H_{\alpha}^{(0) \pm}(x)$ and integrating using Eq. (99), we finally obtain

$$
D_{\alpha}^{(0)}=-\frac{q^{2} u_{\alpha}}{24 r_{0}^{2}} \int_{0}^{\widetilde{\infty}} J_{1}(\lambda) d \lambda=-\frac{q^{2} u_{\alpha}}{24 r_{0}^{2}}
$$

We next turn to calculate $D_{\alpha}^{(1)}$. Writing Eq. 110) for $n=1$ in the form

$$
D_{\alpha}^{(1)}=-q^{2} r_{0} \lim _{\epsilon \rightarrow 0}\left[\left(r_{0} / \epsilon\right) \sum_{l=0}^{\widetilde{\infty}}\left(\epsilon / r_{0}\right) \int_{L \epsilon / r_{0}}^{\widetilde{\infty}} H_{\alpha}^{(1)}(\lambda) d \lambda\right]
$$

and applying the summation formula (111), we obtain

$$
D_{\alpha}^{(1)}=-q^{2} r_{0} \lim _{\epsilon \rightarrow 0}\left[\left(r_{0} / \epsilon\right) \int_{0}^{\widetilde{\infty}} d x \int_{x}^{\widetilde{\infty}} H_{\alpha}^{(1)}(\lambda) d \lambda\right]
$$

with all $O\left(\epsilon^{2}\right)$ terms appearing in Eq. (111) vanishing in the limit $\epsilon \rightarrow 0$. By integrating the last expression by parts with respect to $x$ (noticing the vanishing of the surface term) one finds

$$
D_{\alpha}^{(1)}=-q^{2} r_{0} \lim _{\epsilon \rightarrow 0}\left[\left(r_{0} / \epsilon\right) \int_{0}^{\widetilde{\infty}} x H_{\alpha}^{(1)}(x) d x\right]
$$


Here, the integrand contains only terms of the form $\propto x^{k} J_{n}(x)$, with $k-n$ being positive odd integers [see Eqs. (89) and (92)]. Hence, by virtue of Eq. (99), the integral vanishes, yielding

$$
D_{\alpha}^{(1)}=0 .
$$

Finally, from Eq. (110) with $n=2$ we obtain

$$
D_{\alpha}^{(2)}=-q^{2} r_{0} \lim _{\epsilon \rightarrow 0}\left[\sum_{l=0}^{\widetilde{\infty}}\left(\epsilon / r_{0}\right)\left(L \epsilon / r_{0}\right)^{-1} \int_{L \epsilon / r_{0}}^{\widetilde{\infty}} H_{\alpha}^{(2)}(\lambda) d \lambda\right]=-q^{2} r_{0} \int_{0}^{\widetilde{\infty}}(d x / x) \int_{x}^{\widetilde{\infty}} H_{\alpha}^{(2)}(\lambda) d \lambda,
$$

which, after integrating by parts, becomes

$$
D_{\alpha}^{(2)}=-q^{2} r_{0}\left[\int_{0}^{\widetilde{\infty}} \ln x H_{\alpha}^{(2)}(x) d x-\lim _{x \rightarrow 0}\left(\ln x \int_{x}^{\widetilde{\infty}} H_{\alpha}^{(2)}(\lambda) d \lambda\right)\right] .
$$

We notice here that the second integral on the RHS is just $C_{\alpha}+O(x)$ [up to a multiplicative constant; see Eq. (960)]. The above-deduced vanishing of the parameter $C_{\alpha}$ guarantees the definiteness of the $x \rightarrow 0$ limit in Eq. (123), and makes the second term on its RHS vanish. Note the way the vanishing of the parameter $C_{\alpha}$ appears as a necessary self-consistency condition in our scheme: had we got $C_{\alpha} \neq 0$, the parameter $D_{\alpha}$ would have been indefinite, and the whole regularization scheme would have been rendered meaningless.

As the $x \rightarrow 0$ limit in Eq. (123) vanishes, we are left with

$$
D_{\alpha}^{(2)}=-q^{2} r_{0} \int_{0}^{\widetilde{\infty}} \ln x H_{\alpha}^{(2)}(x) d x .
$$

With the explicit form of $H_{\alpha}^{(2)}$, given in Eqs. (90) and (93), the integrand in the last expression is found to consist of various terms of the form $\propto x^{k} \ln x J_{n}(x)$, with $k-n$ being positive odd integers. The integrals $\int_{0}^{\widetilde{\infty}}$ of such terms can be read from the formula (100), which we derive in Appendix B. Using this formula we obtain expressions for $D_{r}^{(2)}$ and $D_{t}^{(2)}$, which, after substituting for $\dot{r}_{*}, \ddot{r}_{*}, \dddot{r}_{*}, \ddot{s}, \dddot{s}, f_{1}, f_{2}$, and $f_{3}$, read

$$
D_{r}^{(2)}=\frac{1}{3} q^{2}\left(f^{-1} \dddot{r}+\dot{r} \ddot{v} \ddot{u}\right)+\frac{q^{2} \dot{r}}{24 f^{2} r_{0}^{2}}\left[f(4 f-3)+8 r_{0} f^{\prime}\left(f-r_{0} \ddot{r}\right)+2 r_{0}^{2}\left(3 f f^{\prime \prime}-f^{\prime 2}\right)\right],
$$

and

$$
\begin{aligned}
D_{t}^{(2)}= & -\frac{1}{3} q^{2}\left(f \dddot{r}+f^{2} \ddot{t} \ddot{v} \ddot{u}\right)-\frac{1}{2} q^{2} f^{\prime} \ddot{r} \ddot{t} \\
& +\frac{q^{2} \dot{t}}{24 r_{0}^{2}}\left[3 f-4 f^{2}+4 f^{-1}\left(f^{\prime}\right)^{2} r_{0}^{2} \dot{r}^{2}-8 f^{\prime \prime} r_{0}^{2} \dot{r}^{2}-4 f^{\prime} r_{0}^{2} \ddot{r}-2 f f^{\prime \prime} r_{0}^{2}-8 f f^{\prime} r_{0}\right] .
\end{aligned}
$$

We are now in position to write an expression for the "overall" parameter $D_{\alpha}$. We have $D_{\alpha}=D_{\alpha}^{(0)}+D_{\alpha}^{(2)}$, yielding

$$
D_{r}=\frac{1}{3} q^{2}\left(f^{-1} \dddot{r}+\dot{r} \ddot{v} \ddot{u}\right)+\frac{q^{2} \dot{r}}{12 f^{2} r_{0}^{2}}\left[2 f(f-1)+4 r_{0} f^{\prime}\left(f-r_{0} \ddot{r}\right)+r_{0}^{2}\left(3 f f^{\prime \prime}-f^{\prime 2}\right)\right],
$$

and

$$
\begin{aligned}
D_{t}= & -\frac{1}{3} q^{2}\left(f \dddot{r}+f^{2} \ddot{t} \ddot{v} \ddot{u}\right)-\frac{1}{2} q^{2} f^{\prime} \ddot{r} \ddot{t} \\
& +\frac{q^{2} \dot{t}}{12 r_{0}^{2}}\left[2 f(1-f)+2 f-1\left(f^{\prime}\right)^{2} r_{0}^{2} \dot{r}^{2}-4 f^{\prime \prime} r_{0}^{2} \dot{r}^{2}-2 f^{\prime} r_{0}^{2} \ddot{r}-f f^{\prime \prime} r_{0}^{2}-4 f f^{\prime} r_{0}\right] .
\end{aligned}
$$

In the case of radial motion, the four-acceleration's components admit the explicit form $a^{r}=\ddot{r}+\frac{1}{2} f^{\prime}$ and $a^{t}=$ $\ddot{t}+f^{\prime} \dot{r}_{*} \dot{t}$. Recalling also that in the spacetime class considered here the Ricci scalar reads $R=-\left[f^{\prime \prime}+4 f^{\prime} / r+2(f-1) / r^{2}\right]$, one can show that the above two expressions for $D_{r}$ and $D_{t}$ can be put into the simple vectorial form

$$
D_{\alpha}=\frac{1}{3} q^{2}\left(\dot{a}_{\alpha}-a^{2} u_{\alpha}\right)-\frac{1}{12} q^{2} R u_{\alpha} .
$$

Comparing now this result with Eqs. (10) and (11), and recalling that in the case considered here (that of radial motion on static spherically-symmetric background) the first two terms in the expression for $F_{\alpha}^{(\text {Ricci) }}$ [Eq. (11)] cancel out, we arrive at the remarkable conclusion that $D_{\alpha}$ is exactly the "standard" local part of the self-force:

$$
D_{\alpha}=F_{\alpha}^{(\mathrm{ALD})}+F_{\alpha}^{(\mathrm{Ricci})} \text {. }
$$




\section{SUMMARY AND CONCLUDING REMARKS}

The total self force acting on the radially moving scalar particle is obtained by substituting $F_{\alpha}^{(\text {tail })}$ from Eq. (98) in Eq. (9). By virtue of Eq. (130), the contribution of $D_{\alpha}$ to the tail term is then found to exactly cancel out the local term in the expression for the total self force. This, in addition to the vanishing of the parameter $C_{\alpha}$, leads to the simple result

$$
F_{\alpha}^{(\text {total })}=\sum_{l=0}^{\infty}\left(F_{\alpha}^{l \pm}-A_{\alpha}^{ \pm} L-B_{\alpha}\right)
$$

(where, we recall, $L=l+1 / 2$ ). An even simpler form is obtained when calculating $F_{\alpha}^{(\text {total) }}$ using the averaged value of the modes $F_{\alpha}^{l}$, obtained by averaging over their two one-sided values. Then, by virtue of Eq. (102), we find

$$
F_{\alpha}^{(\text {total })}=\sum_{l=0}^{\infty}\left(\bar{F}_{\alpha}^{l}-B_{\alpha}\right)
$$

where $\bar{F}_{\alpha}^{l} \equiv \frac{1}{2}\left(F_{\alpha}^{l+}+F_{\alpha}^{l-}\right)$. Recall that the parameter $B_{\alpha}$, given in Eq. (106), is just the asymptotic value of the averaged $l$-mode $\bar{F}_{\alpha}^{l}$ at the limit $l \rightarrow \infty$; namely, the total self force is obtained by simply subtracting from each (two one-sided averaged) mode its large $l$ asymptotic value, and then summing over all modes.9

To summarize, in this paper we have developed a method for calculating the self force on a scalar particle in curved spacetime, through regularization of the multipole mode sum. The basic difficulty in applying the mode decomposition approach - the apparent divergence of the sum over modes - has been taken care of by the introduction of an appropriate regularization scheme, providing a practical prescription for calculating the self force. It should be emphasized that the proposed method does not involve any weak-field or slow-motion approximations, and thus allows effective calculations of the self force even for strong field orbits.

The basic expression for the tail part of the self force is given in Eq. (98). This expression was developed here for radial motion; however, the same general form applies for any trajectory [29], with the details of the orbit encoded only in the values of the regularization parameters (as well as, of course, in the form of the "bare" modes $F_{\alpha}^{l \pm}$ ). To apply this general expression for a given trajectory requires knowledge of (i) the modes $F_{\alpha}^{l}$, to be derived by supplementary (basically straightforward) numerical analysis, as done in Refs. 23, 24, 26, ; and (ii) four regularization parameters for each spacetime component of the force. In this paper we have worked out the entire calculation of the regularization parameters for the case of radial motion. For any other trajectory, the derivation of these parameters can be carried out along the same lines, based on the explicit form of the Green's function's l-mode given in Eq. (30), with Eqs. (61), 63) and (65). To that end, one first obtains an expression for the gradient of the Green's function's $l$-mode, as in Eq. (70) [supplemented with Eqs. (72) and (73)]. One next expands this gradient in powers of proper time $\tau$ along the worldline about the force's evaluation point, and re-expresses the resulting expansion as an expansion in powers of $1 / L$, by holding $\tau L$ fixed - as in Eq. (87) [supplemented with Eqs. (88)-(93)]. One finally uses the values of the above expansion coefficients [denoted in this paper by $H_{\alpha}^{(n)}(\tau L)$ ] to construct the regularization parameters through Eqs. (96) and (97).

For a scalar particle moving radially in a spacetime of the class considered in this paper, we found the total self force to be given by Eq. (132). This constitutes our main result for the radial motion case, together with the explicit values of the regularization parameters given in Eqs. (102), (106), (108), and (129). We have found that, in the radial motion case, the parameters $\bar{A}_{\alpha}$ and $C_{\alpha}$ both vanish the one-sided values of $A_{\alpha}$ do not vanish; they are given in Eq. (101)], and the parameter $D_{\alpha}$ is just the standard local part of the self force. The vanishing of $C_{\alpha}$, shown here explicitly, appeared as a necessary condition for the definiteness of the whole scheme (had $C_{\alpha} \neq 0$, the parameter $D_{\alpha}$ would have diverge; see the discussion in Sec. VII). This point serves to demonstrate the self-consistency of the regularization scheme.

A question arises, whether the above results (the vanishing of $\bar{A}_{\alpha}$ and $C_{\alpha}$ and the special value of $D_{\alpha}$ ) represent generic features of the regularization scheme, or rather are special to radial trajectories. Preliminary investigation 29] suggests that, indeed, $\bar{A}_{\alpha}$ and $C_{\alpha}$ vanish for all trajectories, at least in the Schwarzschild case. As to the parameter

\footnotetext{
9 The simplicity of our main result, Eq. (132), may lead one to wonder whether there could be simple arguments leading directly to this result. Such arguments might perhaps rely on general properties of the Hadamard expansion. This should make an interesting subject for further investigation.
} 
$D_{\alpha}$, this was shown so far to obey Eq. (130) at least in one more important example, that of a circular orbit around a Schwarzschild black hole [29,22]. It might be conjectured (and be subject to further investigation) that Eq. (130) holds for any trajectory in any static spherically-symmetric background. In that case, the simple Eq. (132) for the total self force would be valid for all such trajectories.

Under the above conjecture, we find that regularization of the total self force requires knowledge of just one parameter, $B_{\alpha}$, representing the asymptotic value of the modes $\bar{F}_{\alpha}^{l}$ as $l \rightarrow \infty$. The value of $B_{\alpha}$ for any specific radial trajectory on any given spherically-symmetric spacetime, can be read from Eq. 106) (valid regardless of the above conjecture). For example, in the special case of a static particle we find $B_{t}^{\text {(static) }}=0$ and

$$
B_{r}^{\text {(static) }}=-\frac{q^{2}}{2 r_{0}^{2}}\left(1+\frac{r_{0} f^{\prime}}{2 f}\right)
$$

For radial geodesic motion we find

$$
B_{t}^{\text {(geodesic) }}=-\frac{q^{2}}{2 r_{0}^{2}} \dot{r} E, \quad B_{r}^{\text {(geodesic) }}=-\frac{q^{2}}{2 r_{0}^{2}}\left(2-E^{2} / f\right),
$$

where $E \equiv-u_{t}$ is the energy parameter (which is a constant of motion in the absence of self force effect). For the value of $B_{r}$ in the case of uniform circular motion in Schwarzschild spacetime (the derivation of which will be presented elsewhere [29]) we refer the reader to Eq. (34) of Ref. [22].

The applicability of the regularization prescription described here was demonstrated recently in actual calculations of the self force for various scenarios. Burko first studied the cases of static [23] and circular [24] orbits in the Schwarzschild spacetime. For these stationary scenarios, the modes $F_{\alpha}^{l}$ were obtained by summing over the Fouriermultipole modes $F_{\alpha}^{l m \omega}$, first derived by solving the appropriate ordinary field equations in the frequency domain. Later, Barack and Burko [26] analyzed the case of radial motion in Schwarzschild. In this case, which is no longer stationary, numerical evolution of the appropriate partial DE in the time domain was applied to directly infer the modes $F_{\alpha}^{l}$. In each of these studies, the overall force acting on the scalar particle was finally deduced by summing over all modes, using the above regularization scheme. In each of the cases analyzed, the vanishing of $C_{\alpha}$ was demonstrated, and the analytically-derived expressions for $A_{\alpha}$ and $B_{\alpha}$ were verified. Aside from demonstrating the applicability of the regularization scheme and providing verification for the values of the regularization parameters, the above studies yielded valuable physical results, as mentioned in the Introduction.

Of course, the analysis of the scalar self force merely serves as a toy model for more realistic cases. Generalization of the regularization scheme to the electromagnetic self force seems possible, based on the existing formalism [7, 11. Such a generalization is necessary, for example, for resolving the interesting question re-raised recently by Hubeny [30], whether a nearly extreme electrically-charged black hole might be overcharged (and its event horizon by destroyed) by throwing in a charged particle: as pointed out by Hubeny, knowledge of the exact radiation reaction effect is crucial for obtaining a definite answer. More difficult to accomplish would be the important generalization of the scheme to the gravitational self force acting on a mass particle.

Finally, it should be mentioned that a closely related approach was recently applied by Lousto 31] for studying the gravitational self force on a mass particle in Schwarzschild spacetime. This approach is also based on the multipole expansion, yet it employs a different regularization method for the mode sum (it is argued that the correct self force can be deduced by applying the zeta-function regularization technique). For the geodesic motion case studied by Lousto, this approach leads to an expression analogous to Eq. (132).

\section{ACKNOWLEDGEMENTS}

I wish to thank Amos Ori for suggesting the basic idea for the regularization scheme, and for his assistance in developing it. I would also like to thank Lior Burko for discussions and for reading the manuscript.

\section{APPENDIX A: MULTIPOLE EXPANSION OF THE GREEN'S FUNCTION USING "TILDE-SUMMATION"}

DeWitt and Brehme wrote a general expression for the scalar Green's function in curved spacetime [see Eq. (2.21) of Ref. [7]], of the form

$$
G\left(x^{\mu} ; x^{\prime \mu}\right)=a\left(x^{\mu} ; x^{\prime \mu}\right) \delta(\sigma)+b\left(x^{\mu} ; x^{\prime \mu}\right) \Theta(\sigma)
$$


Here, $\sigma$ is plus or minus half the squared geodesic distance between the source point $x^{\mu}$ and the evaluation point $x^{\prime \mu}$, according to whether the geodesic connecting the points (along which the invariant distance is measured) is timelike $(\sigma>0)$ or else $(\sigma \leq 0)$; $a$ is a certain function having a well defined value at $\sigma=0$; and $b$ is a function which may be written as a Taylor expansion in $\sigma$ about $\sigma=0$. [This expansion was shown by Hadamard (see pp. 96-98 in Ref. [8]) to converge uniformly at least inside the region where $\sigma$ is single valued.] While the first term in Eq. (A1) is associated with the familiar delta function exhibited already in flat spacetime, the second term represents a curvature-induced tail, which "fills" the light cone (defined by $\sigma=0$ ). Note that the Green's function is strongly irregular along the light cone of the source point $x^{\prime \mu}$.

Now, the question to consider is whether the above Green's function may be expanded in terms of the standard spherical harmonic functions $Y^{l m}(\theta, \varphi)$ on a sphere of constant $r, t$. Standard theorem (see, e.g., Ref. [32], p. 513) states that a sufficient condition for (absolute and uniform) convergence of the spherical harmonic expansion is the expanded function being $C^{2}$ on the sphere. This condition is not satisfied here, as the Green's function diverges along the curve generated by the intersection of the future light cone of $x^{\prime \mu}$ and the sphere of constant $r, t$. Therefore, it is not guaranteed, in advance, that such an expansion could be naively applied. Indeed, already in flat spacetime the attempt to apply the multipole expansion to the Green's function turns out to yield a divergent sum - see Eq. (14) and the discussion proceeding it in Sec. III.

Let us introduce the "modified" Green's function (which is not a "Green's function" for the scalar field anymore), defined by

$$
G_{\bmod }=G-\delta G
$$

where

$$
\delta G \equiv a_{0} \delta(\sigma)-b_{0} \Theta(-\sigma),
$$

with $a_{0} \equiv a(\sigma=0)$ and $b_{0} \equiv b(\sigma=0)$. The function $G_{\text {mod }}$ has two essential features: (i) its support inside the light cone is identical to that of the Green's function $G$ (as $\delta G$ has no support there), and (ii) it is continuous throughout any sphere of constant $r, t$. The first feature implies that we can use $G_{\text {mod }}$ instead of $G$ in calculating the self force: We may re-write Eq. (13) as

$$
F_{\alpha}^{(\epsilon)} \equiv q^{2} \int_{-\infty}^{-\epsilon}\left\{G_{\bmod }\left[x_{0}^{\mu} ; x_{p}^{\mu}(\tau)\right]\right\}_{, \alpha} d \tau
$$

as the $\delta G$ term in $G_{\text {mod }}$ contributes nothing to the integral along the particle's worldline.

The second of the above features of $G_{\text {mod }}$, its continuity, may imply that the multipole expansion could now be applied to it. Strictly speaking, $G_{\text {mod }}$ does not satisfy the above sufficient condition for absolute and uniform convergence of the mode sum (i.e., being $C^{2}$ on the sphere); yet, we shall assume here that $G_{\text {mod }}$, being continuous, is already regular enough to admit a convergent mode sum. The results of our analysis turn out to be consistent with this assumption, as the mode-sum of $G$ considered (based on the function $G_{\text {mod }}$ through the use of "tilde summation" - see below) is found to be (absolutely) convergent. The validity of this assumption can also be demonstrated in the flat space case: the $\propto b_{0}$ tail term vanishes in this case, and by expanding the $\delta(\sigma)$ term in Eq. (A3) in spherical harmonics one can easily verify that the $l$-mode of $\delta G$ is exactly the $l$-mode of $G$, given in Eq. (14). The $l$-mode of $G_{\text {mod }}$ then vanishes, and the mode sum converges. Although trivial, this flat-space example may serve to demonstrate how the subtraction of $\delta G$ from $G$ already removes the divergent piece from the $l$-mode, making the mode sum converge. 10 We thus expand $G_{\text {mod }}$ as

$$
G_{\text {mod }}=\sum_{l=0}^{\infty} G_{\text {mod }}^{l}=\sum_{l=0}^{\infty}\left(G^{l}-\delta G^{l}\right),
$$

where $G_{\text {mod }}^{l}$ and $\delta G^{l}$ are the spherical harmonic modes of $G_{\text {mod }}$ and $\delta G$, respectively (obtained by summing over all azimuthal numbers $m$ ).

To proceed, let us now define the new operation $\widetilde{\lim }_{l \rightarrow \infty}$ ("tilde-limit") as follows: Consider a series of numbers $A_{l}$ (with $l=0,1, \ldots, \infty)$. Let $B_{l}^{(j)}$ be any expression of the form

\footnotetext{
${ }^{10}$ One may similarly construct a more sophisticated function $\delta G$, designed to yield a $C^{2}$ modified function $G_{\text {mod }}$ [by canceling also the $O(\sigma)$ and $O\left(\sigma^{2}\right)$ terms in the Taylor expansion of $\left.b\right]$ being sufficiently regular to assure uniform and absolute convergence of the multipole expansion, by standard mathematical theorem. Such an improved construction will not be examined here.
} 


$$
B_{l}^{(j)}=a_{j} l^{b_{j}} \cos \left(\alpha_{j} l+\beta_{j}\right)
$$

where $a_{j}, b_{j}, \alpha_{j}$, and $\beta_{j}$ are some $l$-independent real numbers, with $\alpha_{j} \neq 0$ for all $j$. If there exists a finite number $k$ of expressions $B_{l}^{(j)}$ of this form (with $j=1,2, \ldots, k$ ), such that subtracting their sum from the original series $A_{l}$ would yield a well-defined finite limit as $l \rightarrow \infty$, then we define the "tilde-limit" $\widetilde{\lim }_{l \rightarrow \infty} A_{l}$ as in Eq. 16 ).

One may easily be convinced that the tilde-limit is single-valued (when existing). For, suppose that for a given series $A_{l}$ there were two different sets of quantities $B_{l}^{(j)}$, one (denoted by $\bar{B}_{l}^{(\bar{j})}$ ) yielding $\widetilde{\lim }_{l \rightarrow \infty} A_{l}=\bar{c}$, and the other (denoted by $\hat{B}_{l}^{(\hat{j})}$ ) yielding $\widetilde{\lim }_{l \rightarrow \infty} A_{l}=\hat{c} \neq \bar{c}$. Then, for the difference between the two limits one would have found $\lim _{l \rightarrow \infty}\left[\sum_{\hat{j}} \hat{B}_{l}^{(\hat{j})}-\sum_{\bar{j}} \bar{B}_{l}^{(\bar{j})}\right]=\hat{c}-\bar{c} \neq 0$. This, however, is impossible, as the (standard) limit $l \rightarrow \infty$ of any quantity of the type $B_{l}^{(j)}$ is either diverging or zero, and so is the limit of any finite sum of such quantities. Hence, we must have $\hat{c}=\bar{c}$, and the tilde-limit is single valued. In particular, we find that if there exists a finite standard limit $\lim _{l \rightarrow \infty} A_{l}$, then $\lim _{l \rightarrow \infty} A_{l}=\widetilde{\lim }_{l \rightarrow \infty} A_{l}$.

We can now also define the "tilde-sum" of a series $A_{l}$, as in Eq. (17). Again, if the "tilde-sum" of a series exists, then it is unique. Also, if the standard sum $\sum_{l \rightarrow \infty} A_{l}$ converges, then we may replace it with a "tilde-sum" operation. In particular, we may replace the convergent standard sum of Eq. A5 with a tilde-summation:

$$
G_{\text {mod }}=\sum_{l=0}^{\widetilde{\infty}}\left(G^{l}-\delta G^{l}\right) .
$$

Below we show that

$$
\sum_{l=0}^{\widetilde{\infty}} \delta G^{l}=0
$$

(for any evaluation point $x_{0}^{\mu}$ lying inside the future light cone of the source point $x_{p}^{\mu}$ ). As a consequence, the tilde-sum of $G^{l}$ is found to be finite and equal to $G_{\bmod }$-as indicated in Eq. (18) of Sec. III.

Combining Eqs. (A4) and (18) we conclude that the self-force can be calculated by analyzing the modes $G^{l}$ of the original Green's function, provided that in order to sum over all modes one applies the tilde-summation instead of the standard summation. The validity of this statement crucially depends on the vanishing of the tilde-sum over $\delta G^{l}$ [Eq. (A8)], which we now prove.

\section{Proof of Eq. (A8)}

To calculate the $l$-modes of $\delta G$, it is convenient to use a spherical coordinate system in which the source point $x_{\mathrm{p}}^{\mu}$ lies on the polar axis (i.e., $\theta_{p}=0$ ). In this coordinate system, contributions to $\delta G$ would come only from the $m=0$ modes:

$$
\begin{aligned}
\delta G^{l}= & \sum_{m=-l}^{l} Y_{l m}(\theta, \varphi) \int_{0}^{2 \pi} d \varphi^{\prime} \int_{-1}^{1} d\left(\cos \theta^{\prime}\right) \delta G\left(\sigma^{\prime}\right) Y_{l m}^{*}\left(\theta^{\prime}, \varphi^{\prime}\right)= \\
& L \int_{-1}^{1} d\left(\cos \theta^{\prime}\right)\left[a_{0} \delta\left(\sigma^{\prime}\right)-b_{0} \Theta\left(-\sigma^{\prime}\right)\right] P_{l}(\cos \theta) P_{l}\left(\cos \theta^{\prime}\right)
\end{aligned}
$$

where the integration is carried out over a sphere spanned by $\theta^{\prime}, \varphi^{\prime}$ (containing the evaluation point $\left.x^{\mu}\right), L \equiv(l+1 / 2)$, and $\sigma^{\prime}$ is half the squared geodesic distance between the source point and the integration point $\theta^{\prime}, \varphi^{\prime}$. Now, the future light cone of the source point (along which $\sigma^{\prime}=0$ ) intersects the integration sphere along a circle $\theta^{\prime}=$ const $\equiv \theta_{0}$. The $\delta\left(\sigma^{\prime}\right)$ term in Eq. (A9) contributes to the integration only along this circle, while the $\Theta(-\sigma)$ term contributes only across the part of the sphere outside it. We thus find

$$
\delta G^{l}=L P_{l}(\cos \theta)\left[\hat{a}_{0} P_{l}\left(\cos \theta_{0}\right)-b_{0} \int_{-1}^{\cos \theta_{0}} P_{l}\left(\cos \theta^{\prime}\right) d\left(\cos \theta^{\prime}\right)\right] \equiv \delta G_{(\delta)}^{l}+\delta G_{(\Theta)}^{l},
$$

where $\hat{a}_{0} \equiv a_{0} /\left|d \sigma^{\prime} / d\left(\cos \theta^{\prime}\right)\right|_{\theta^{\prime}=\theta_{0}}$ is a constant, and where the symbols $\delta G_{\delta}^{l}$ and $\delta G_{\Theta}^{l}$ represent the two terms proportional to $\hat{a}_{0}$ and $b_{0}$, respectively. 
To calculate the tilde-sum over $\delta G^{l}$ we make use of the finite-sum identity [referred to as "Christoffel's first summation formula" - see, e.g., Eq. 3.8(20) of Ref. [33]]

$$
(x-y) \sum_{l^{\prime}=0}^{l} L P_{l^{\prime}}(x) P_{l^{\prime}}(y)=\frac{1}{2}(l+1)\left[P_{l+1}(x) P_{l}(y)-P_{l+1}(y) P_{l}(x)\right],
$$

valid for $|x| \leq 1$ and $|y| \leq 1$. Applying this formula to Eq. (A10), we obtain

$$
\begin{aligned}
\sum_{l=0}^{\widetilde{\infty} \delta G^{l}=} & \widetilde{\lim }_{l \rightarrow \infty} \sum_{l^{\prime}=0}^{l}\left(\delta G_{(\delta)}^{l^{\prime}}+\delta G_{(\Theta)}^{l^{\prime}}\right)= \\
& \frac{1}{2} \hat{a}_{0} \widetilde{\lim }_{l \rightarrow \infty}\left[(l+1) \frac{P_{l+1}(x) P_{l}\left(x_{0}\right)-P_{l+1}\left(x_{0}\right) P_{l}(x)}{x-x_{0}}\right] \\
& -\frac{1}{2} b_{0} \widetilde{\lim }_{l \rightarrow \infty}\left[(l+1) \int_{-1}^{x_{0}} \frac{P_{l+1}(x) P_{l}\left(x^{\prime}\right)-P_{l+1}\left(x^{\prime}\right) P_{l}(x)}{x-x^{\prime}} d x^{\prime}\right],
\end{aligned}
$$

where $x$ and $x_{0}$ stand for $\cos \theta$ and $\cos \theta_{0}$. (Recall that for any evaluation point $x^{\mu}$ lying inside the future light cone of the source point $x_{p}^{\mu}$, we have $\cos \theta>\cos \theta_{0} \geq \cos \theta^{\prime}$; hence, the denominators appearing in the last expression are strictly positive.)

Let us consider first the tilde-sum of $\delta G_{(\delta)}^{l}$. For values of $\theta$ satisfying $\epsilon \leq \theta \leq \pi-\epsilon$ (where $\epsilon>0$ ) we have the large-l asymptotic form

$$
P_{l}(\cos \theta) \propto \sqrt{2}(l \pi \sin \theta)^{-1 / 2} \cos (L \theta+\pi / 4)+O\left(l^{-3 / 2}\right)
$$

[see, e.g., Eq. 3.9(2) of Ref. [33]]. Using this asymptotic form with Eq. (A12), we may easily write $\sum_{l^{\prime}=0}^{l} \delta G_{(\delta)}^{l^{\prime}}$ as a sum of a few terms of the form $a_{j} \cos \left(\alpha_{j} l+\beta_{j}\right)+O\left(l^{-1}\right)$ (in the case $\left.0<\theta<\pi\right)$, or of the form $a_{j} l^{1 / 2} \cos \left(\alpha_{j} l+\beta_{j}\right)+O\left(l^{-1 / 2}\right.$ ) (in the case $\theta=0$ or $\pi$ ), where $a_{j}, \alpha_{j} \neq 0$, and $\beta_{j}$ are certain functions of $\theta$ and $\theta_{0}$ (independent of $l$ ). Such terms all vanish at the tilde-limit $l \rightarrow \infty$. Thus, we clearly have

$$
\sum_{l=0}^{\widetilde{\infty}} \delta G_{(\delta)}^{l}=0
$$

We next turn to calculate the tilde-sum of $\delta G_{(\Theta)}^{l}$. Integrating by parts in Eq. (A12), and using

$$
\int P_{l}(x) d x=\frac{P_{l+1}(x)-P_{l-1}(x)}{2 l+1}
$$

[see Eq. 7.111 of Ref. [27], together with Eq. 8.733-4 therein], we obtain

$$
\begin{aligned}
\sum_{l^{\prime}=0}^{l} \delta G_{(\Theta)}^{l^{\prime}}=-\frac{1}{2} b_{0}(l+1)\{ & \frac{P_{l+1}(x)}{2 l+1}\left[\left.\frac{P_{l+1}\left(x^{\prime}\right)-P_{l-1}\left(x^{\prime}\right)}{x-x^{\prime}}\right|_{-1} ^{x_{0}}-\int_{-1}^{x_{0}} \frac{P_{l+1}\left(x^{\prime}\right)-P_{l-1}\left(x^{\prime}\right)}{\left(x-x^{\prime}\right)^{2}} d x^{\prime}\right] \\
& \left.-\frac{P_{l}(x)}{2 l+3}\left[\left.\frac{P_{l+2}\left(x^{\prime}\right)-P_{l}\left(x^{\prime}\right)}{x-x^{\prime}}\right|_{-1} ^{x_{0}}-\int_{-1}^{x_{0}} \frac{P_{l+2}\left(x^{\prime}\right)-P_{l}\left(x^{\prime}\right)}{\left(x-x^{\prime}\right)^{2}} d x^{\prime}\right]\right\} .
\end{aligned}
$$

The surface terms here vanish at the lower boundary, as $P_{l+1}(-1)=P_{l-1}(-1)$ and $P_{l+2}(-1)=P_{l}(-1)$ [recalling $P_{l}(-1)=(-1)^{l}$ ]. The contribution from the upper boundary dies off at large $l$ [by virtue of Eq. (A13)] as $\propto l^{-1}$ (times oscillations) for $\theta \neq 0$, or as $\propto l^{-1 / 2}$ (times oscillations) for $\theta=0$. In both cases, this contribution thus vanishes at the standard limit $l \rightarrow \infty$, and hence also at the tilde-limit. Consider next the integral terms: The difference between two Legendre functions appearing in these terms may be globally bounded (in absolute value) as

$$
\left|P_{l+1}(\cos \theta)-P_{l-1}(\cos \theta)\right| \leq C_{0}[\pi(l-1)]^{-1 / 2},
$$

where $C_{0}$ is a number independent of $l$ and $\theta$ [see Eq. 8.838 of Ref. [27]. For any $\theta$ and $\theta_{0}$, the integrals of Eq. (A16) are thus each bounded (in absolute value) by $C_{1}\left(\theta, \theta_{0}\right) \times l^{-1 / 2}$, and we find

$$
\left|\sum_{l^{\prime}=0}^{l} \delta G_{(\Theta)}^{l^{\prime}}\right| \leq C_{2}\left(\theta, \theta_{0}\right) l^{-1 / 2}\left[\left|P_{l+1}(\cos \theta)\right|+\left|P_{l}(\cos \theta)\right|\right] \rightarrow 0
$$


as $l \rightarrow \infty$ (the above coefficients $C_{1}$ and $C_{2}$ are $l$-independent). Thus, the standard infinite sum over $\delta G_{(\Theta)}^{l}$ vanishes, and hence also the tilde-sum:

$$
\sum_{l=0}^{\widetilde{\infty} \delta G_{(\Theta)}^{l}}=0
$$

With Eqs. (A14) and A19), Eq. (A8) is verified.

\section{APPENDIX B: DERIVATION OF INTEGRALS}

In this appendix we obtain the tilde-integrals given in Eqs. (99) and (100), which are needed in the calculation of the regularization parameters. We start with Eq. (99). Let $I_{n}^{k}(\lambda)$ denote the primitive function of $\lambda^{k} J_{n}(\lambda)$, where $\lambda$ is a real variable, $J_{n}$ is the Bessel function of the first kind, of order $n$, and $k, n \in \mathbb{N}$ :

$$
I_{n}^{k}(\lambda) \equiv \int \lambda^{k} J_{n}(\lambda) d \lambda
$$

Let also $\widetilde{I}_{n}^{k}$ stand for the definite integral

$$
\widetilde{I}_{n}^{k} \equiv \widetilde{\lim }_{\lambda \rightarrow \infty} \int_{0}^{\lambda}\left(\lambda^{\prime}\right)^{k} J_{n}\left(\lambda^{\prime}\right) d \lambda^{\prime} \equiv \int_{0}^{\widetilde{\infty}} \lambda^{k} J_{n}(\lambda) d \lambda .
$$

Consider first the case $k=0$. The standard integral $\int_{0}^{\infty} J_{n}(\lambda) d \lambda=1$ is well defined and finite. Thus, in this case, the tilde-integration in Eq. (B2) may be replaced with a standard integration, yielding

$$
\widetilde{I}_{n}^{k=0}=1, \quad \forall n \geq 0 .
$$

Consider next the case $k>0$. Writing in Eq. (B1) $\lambda^{k} J_{n}(\lambda)=\lambda^{k-n-1}\left[\lambda^{n+1} J_{n}(\lambda)\right]=\lambda^{k-n-1}\left[\lambda^{n+1} J_{n+1}(\lambda)\right]^{\prime}[$ where use is made of Eq. (66) and a prime denotes $d / d \lambda]$, and integrating by parts, we arrive at the recursive formula

$$
I_{n}^{k}(\lambda)=\lambda^{k} J_{n+1}(\lambda)-(k-n-1) I_{n+1}^{k-1}(\lambda) .
$$

If $0<k \leq n$, then by $k$ successive applications of this recursive formula we obtain

$$
I_{n}^{k}(\lambda)=\sum_{j=0}^{k-1}\left[\frac{(n-k-1+2 j) ! !}{(n-k-1) ! !} \lambda^{k-j} J_{n+1+j}(\lambda)\right]+\frac{(n+k-1) ! !}{(n-k-1) ! !} I_{n+k}^{0}(\lambda),
$$

Now, the Bessel functions $J_{n}(\lambda)$ admit the asymptotic form

$$
J_{n}(\lambda \rightarrow \infty) \sim(2 / \pi \lambda)^{1 / 2} \cos (\lambda-n \pi / 2-\pi / 4)
$$

(see, e.g., Eq. 8.451-1 in [27]). Therefore, each of the $k$ terms in the sum over $j$ in Eq. (B5) diverges at large $\lambda$ as some positive (half-integer) power of $\lambda$ times oscillations with respect to $\lambda$. Clearly, all such terms are eliminated when the tilde-limit $\lambda \rightarrow \infty$ is taken. Also, all of these terms vanish at $\lambda=0$. We are thus left with

$$
\widetilde{I}_{n}^{k}=\frac{(n+k-1) ! !}{(n-k-1) ! !} \widetilde{I}_{n+k}^{0}=\frac{(n+k-1) ! !}{(n-k-1) ! !}, \quad \text { for } 0<k \leq n,
$$

where the last equality is due to Eq. (B3).

If $k>n$ and the difference $k-n$ is even, then by $p \equiv(k-n) / 2$ applications of the recursive formula (B4) we obtain

$$
I_{n}^{k}(\lambda)=\sum_{j=0}^{p-1}(-1)^{j} \frac{(k-n-1) ! !}{(k-n-1-2 j) ! !} \lambda^{k-j} J_{n+1+j}(\lambda)+(-1)^{p}(k-n-1) ! ! I_{(k+n) / 2}^{(k+n) / 2}(\lambda) .
$$

Again, all terms in the sum over $j$ vanish at the tilde-limit $\lambda \rightarrow \infty$ and at $\lambda=0$, leading to 


$$
\begin{aligned}
\widetilde{I}_{n}^{k}= & (-1)^{p}(k-n-1) ! ! I_{(k+n) / 2}^{(k+n) / 2}(\lambda)= \\
& (-1)^{(k-n) / 2}(k-n-1) ! !(k+n-1) ! !, \quad \text { for even } k-n>0,
\end{aligned}
$$

where the last equality is due to Eq. (B7).

The situation is different in case $k>n$ and the difference $k-n$ is odd. Then, following $q=(k-n-1) / 2$ applications of the recursive formula (B4) one obtains

$$
\begin{aligned}
I_{n}^{k}(\lambda)= & \sum_{j=0}^{q-1}(-1)^{j} \frac{(k-n-1) ! !}{(k-n-1-2 j) ! !} \lambda^{k-j} J_{n+1+j}(\lambda)+(-1)^{q}(k-n-1) ! ! I_{(k+n-1) / 2}^{(k+n+1) / 2}= \\
& \sum_{j=0}^{q}(-1)^{j} \frac{(k-n-1) ! !}{(k-n-1-2 j) ! !} \lambda^{k-j} J_{n+1+j}(\lambda),
\end{aligned}
$$

with no residual integral [notice that when Eq. (B4) is applied with the upper index of $I_{n}^{k}$ greater by 1 than its lower index, then the second term on the RHS of this recursive formula vanishes]. This leads, when applying the tilde-limit, to

$$
\widetilde{I}_{n}^{k}=0, \quad \text { for odd } k-n>0 .
$$

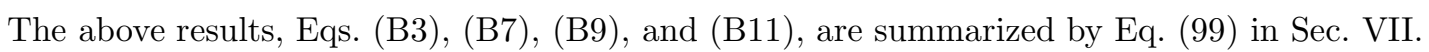

We further need now to calculate the integral given in Eq. (100). Integrating by parts, we express the primitive function of the integrand, $\lambda^{k} J_{n}(\lambda) \ln \lambda$, for odd $k-n>0$, as

$$
I_{n}^{k(\log )} \equiv \int \lambda^{k} J_{n}(\lambda) \ln \lambda d \lambda=I_{n}^{k}(\lambda) \ln \lambda-\int\left[I_{n}^{k}(\lambda) / \lambda\right] d \lambda
$$

By virtue of Eq. (B10), the surface term on the RHS here is dominated at small $\lambda$ by $\propto \lambda^{(k+n+1) / 2} J_{(k+n+1) / 2}(\lambda) \ln \lambda \propto$ $\lambda^{k+n+1} \ln \lambda$, and therefore it vanishes at the limit $\lambda \rightarrow 0$. However, this surface term diverges at the tilde-limit $\lambda \rightarrow \infty$ (as $\lambda^{k-1 / 2} \ln \lambda$ times oscillations with respect to $\lambda$ ). This can be avoided by slightly modifying the definition of a function's tilde-limit, by allowing the quantities $B_{l}^{(j)}$ in Eq. (A6) to also admit the form $B_{l}^{(j)}=a_{j} l^{b_{j}} \ln l \cos \left(\alpha_{j} l+\beta_{j}\right)$ (with $\alpha_{j} \neq 0$ for all $j$ ). It can be shown that all features and results discussed in Appendix A concerning the tilde-limit remain valid also under this wider definition. With the revised definition of the tilde-limit, the surface term in Eq. (B12) vanishes at the tilde-limit $\lambda \rightarrow \infty$ as well as for $\lambda \rightarrow 0$. Denoting $\tilde{I}_{n}^{k(\log )} \equiv \widetilde{\lim }_{\lambda \rightarrow \infty} I_{n}^{k(\log )}$, we then have

$$
\tilde{I}_{n}^{k(\log )}=-\int_{0}^{\widetilde{\infty}}\left[I_{n}^{k}(\lambda) / \lambda\right] d \lambda=-\sum_{j=0}^{(k-n-1) / 2}(-1)^{j} \frac{(k-n-1) ! !}{(k-n-1-2 j) ! !} \widetilde{I}_{n+1+j}^{k-j-1},
$$

where we have substituted for $I_{n}^{k}(\lambda)$ from Eq. (B10). Provided that $k-n$ is an odd number, the difference between the upper and lower indices of $\widetilde{I}_{n+1+j}^{k-j-1}$ is also an odd number, $k-n-2-2 j$. Therefore, by virtue of Eq. (B11), we have $\widetilde{I}_{n+1+j}^{k-j-1}=0$ for any $j$ satisfying $k-j-1>n+1+j$, i.e., $j<(k-n-2) / 2$. We find that of all the terms summed up in Eq. (B13), the only nonvanishing one is the one with $j=(k-n-1) / 2$. Hence,

$$
\tilde{I}_{n}^{k(\log )}=-(-1)^{(k-n-1) / 2}(k-n-1) ! ! \widetilde{I}_{(k+n+1) / 2}^{(k+n-1) / 2}=(-1)^{(k-n+1) / 2}(k-n-1) ! !(k+n-1) ! !,
$$

where, in the last equality, the value of $\widetilde{I}_{(k+n+1) / 2}^{(k+n-1) / 2}$ has been inferred from Eq. (B7). This proves Eq. (100).

[1] B. F. Schutz, Class. Quantum Grav. 16, A131 (1999).

[2] See, e.g., M. Davis et al., Phys. Rev. Lett. 27, 1446 (1971); 28, 1352 (1972); S. A. Teukolsky and W. H. Press, Astrophys. J. 193, 443 (1974); E. Poisson, Phys. Rev. D 47, 1497 (1993); M. Shibata, Prog. Theor. Phys. 90, 595 (1993); B. R. Iyer and C. M. Will, Phys. Rev. Lett. 70, 113 (1993); C. Cutler, D. Kennefick, and E. Poisson, Phys. Rev. D 50, 3816 (1994); E. Poisson and M. Sasaki, ibid. 51, 5753 (1995); S. W. Leonard and E. Poisson, ibid. 56, 4789 (1997). 
[3] A. G. Wiseman, Phys. Rev. D 61, 084014 (2000).

[4] L. M. Burko, Importance of conservative self forces for binaries undergoing radiation damping (in preparation).

[5] P. A. M. Dirac, Proc. R. Soc. London A167, 148 (1938).

[6] D. V. Gal'tsov, J. Phys. A 15, 3737 (1982).

[7] B. S. DeWitt and R. W. Brehme, Ann. Phys. (N.Y.) 9, 220 (1960).

[8] J. Hadamard, Lectures on Cauchy's Problem in Linear Partial Differential Equations (Dover, New York, 1952).

[9] Y. Mino, M. Sasaki, and T. Tanaka, Phys. Rev. D 55, 3457 (1997).

[10] Y. Mino, Prog. Theor. Phys. 99, 79 (1998); Y. Mino and H. Nakano, ibid. 100, 507 (1998).

[11] T. C. Quinn and R. M. Wald, Phys. Rev. D 56, 3381 (1997).

[12] J. M. Hobbs, Ann. Phys. (N.Y.) 47, 141 (1968).

[13] T. C. Quinn, Phys. Rev. D (to be published), gr-qc/0005030.

[14] T. C. Quinn and R. M. Wald, Phys. Rev. D 60, 064009 (1999).

[15] C. M. DeWitt and B. S. DeWitt, Physics (N.Y.) 1, 3 (1964).

[16] A. G. Smith and C. M. Will, Phys. Rev. D 22, 1276 (1980).

[17] V. P. Frolov and A. I. Zel'nikov in Abstracts of Contributed Papers, Ninth International Conference GRG, 1980, Vol. 3, p. 555 .

[18] V. P. Frolov and A. I. Zel'nikov, Zh. Éksp. Teor. Fiz. 82, 321 (1982) [Sov. Phys. JETP 55, 191 (1982)].

[19] E. T. Copson, Proc. R. Soc. London A188, 184 (1928).

[20] B. Linet, J. Phys. A 9, 1081 (1976).

[21] A. Ori, Phys. Lett. A 202, 347 (1995); Phys. Rev. D 55, 3444 (1997).

[22] L. Barack and A. Ori, Phys. Rev. D 61, 061502(R) (2000).

[23] L. M. Burko, Class. Quantum Grav. 17, 227 (2000).

[24] L. M. Burko, Phys. Rev. Lett. D 84, 4529 (2000).

[25] L. M. Burko, in Proceedings of the 3rd Amaldi Conference on Gravitational Waves, edited by Sydney Meshkov, grqc/9911089

[26] L. Barack and L. M. Burko, Phys. Rev. D (to be published), gr-qc/0007033.

[27] I. S. Gradshteyn and I. M. Ryzhik, Tables of Integrals, Series and Products (Academic, New York, 1980).

[28] See, for example, Eq. (3.62) in J. D. Jackson, Classical Electrodynamics, 3rd ed. (John Wiley, New York, 1999).

[29] A. Ori (unpublished).

[30] V. E. Hubeny, Phys. Rev. D 59, 064013 (1999).

[31] C. O. Lousto, Phys. Rev. Lett. 84, 5251 (2000).

[32] R. Courant and D. Hilbert, Methods of Mathematical Physics (Interscience Publishers, New York, 1953), Vol. I.

[33] A. Erdélyi et al., Higher Transcendental Functions (McGraw-Hill, New York, 1953), Vol. 1. 